\title{
Myeloid-specific Asx/2 deletion limits diet-induced obesity by regulating energy expenditure
}

\author{
Wei Zou, ${ }^{1}$ Nidhi Rohatgi, ${ }^{1}$ Jonathan R. Brestoff, ${ }^{1}$ John R. Moley, ${ }^{1}$ Yongjia Li, ${ }^{1}$ Jesse W. Williams, ${ }^{1}$ Yael Alippe, ${ }^{2}$ Hua Pan, ${ }^{3}$ \\ Terri A. Pietka, ${ }^{4}$ Gabriel Mbalaviele, ${ }^{2}$ Elizabeth P. Newberry, ${ }^{5}$ Nicholas O. Davidson, ${ }^{5}$ Anwesha Dey, ${ }^{6}$ Kooresh I. Shoghi, ${ }^{7,8,9}$ \\ Richard D. Head, ${ }^{10}$ Samuel A. Wickline, ${ }^{3}$ Gwendalyn J. Randolph, ${ }^{1}$ Nada A. Abumrad, ${ }^{4}$ and Steven L. Teitelbaum ${ }^{1,2,11}$ \\ 'Department of Pathology and Immunology and 'Division of Bone and Mineral Diseases, Department of Medicine, Washington University School of Medicine, St. Louis, Missouri, USA. ${ }^{3}$ Department \\ of Cardiovascular Sciences, Morsani College of Medicine, University of South Florida, Tampa, Florida, USA. ${ }^{4}$ Division of Ceriatrics and Nutritional Science, Department of Medicine, and ${ }^{5}$ Division of \\ Gastroenterology, Department of Medicine, Washington University School of Medicine, St. Louis, Missouri, USA. ${ }^{6}$ Department of Discovery Oncology, Genentech Inc., South San Francisco, California, USA. \\ ${ }^{7}$ Department of Radiology, ${ }^{8}$ Department of Biomedical Engineering, ${ }^{9}$ Division of Biology and Biomedical Sciences and ${ }^{10}$ Department of Cenetics, Washington University School of Medicine, St. Louis, \\ Missouri, USA. "Shriners Hospitals for Children, St. Louis, Missouri, USA.
}

\begin{abstract}
We previously established that global deletion of the enhancer of trithorax and polycomb (ETP) gene, Asx/2, prevents weight gain. Because proinflammatory macrophages recruited to adipose tissue are central to the metabolic complications of obesity, we explored the role of ASXL2 in myeloid lineage cells. Unexpectedly, mice without Asx/2 only in myeloid cells (Asx/2 ${ }^{\mathrm{LLySM}}$ ) were completely resistant to diet-induced weight gain and metabolically normal despite increased food intake, comparable activity, and equivalent fecal fat. Asx $2^{\mathrm{ALysM}}$ mice resisted HFD-induced adipose tissue macrophage infiltration and inflammatory cytokine gene expression. Energy expenditure and brown adipose tissue metabolism in Asx/2 ${ }^{\mathrm{LLysM}}$ mice were protected from the suppressive effects of HFD, a phenomenon associated with relatively increased catecholamines likely due to their suppressed degradation by macrophages. White adipose tissue of HFD-fed Asx/2 ${ }^{\Lambda \mathrm{Lys} M}$ mice also exhibited none of the pathological remodeling extant in their control counterparts. Suppression of macrophage $A s x / 2$ expression, via nanoparticlebased siRNA delivery, prevented HFD-induced obesity. Thus, ASXL2 controlled the response of macrophages to dietary factors to regulate metabolic homeostasis, suggesting modulation of the cells' inflammatory phenotype may impact obesity and its complications.
\end{abstract}

\section{Introduction}

Obesity is characterized by chronic low-grade inflammation and macrophage accumulation in white adipose tissue (WAT). These cells regulate metabolism by producing proinflammatory cytokines, such as TNF- $\alpha$, that compromise glucose homeostasis (1). The concept of macrophage-mediated inflammation impairing adipose tissue health is fortified by the observation that macrophages in WAT of obese patients generally polarize to the M1 phenotype, while lean fat contains an abundance of M2 cells (2). This concept raises the possibility that promoting a predominance of hypoinflammatory macrophages in patients may reduce pathological remodeling of WAT and as such, diminish the complications of obesity. In fact, ablation of CD11c-expressing cells normalizes insulin sensitivity in obese mice (3). Absence of proinflammatory macrophages, however, does not influence body weight, underscoring the uncertainty of the cell's precise effect on obesity.

Authorship note: WZ and NR are co-first authors.

Conflict of interest: SAW is equity holder in Trasir Therapeutics, Inc. GM is a consultant for Aclaris Therapeutics, Inc.

Copyright: ( 2020 , American Society for Clinical Investigation.

Submitted: March 11, 2019; Accepted: February 4, 2020; Published: April 20, 2020

Reference information: J Clin Invest. 2020;130(5):2644-2656.

https://doi.org/10.1172/JCl128687.
We previously reported that global deletion of the enhancer of trithorax and polycomb (ETP) gene, Asxl2, completely protects mice from HFD-induced weight gain while inducing lipodystrophy and hepatic steatosis (4). Reasoning that this phenotype is likely mediated by altered function of metabolic tissues, specifically hepatocytes and adipocytes, we conditionally deleted the gene in liver or fat. Unlike their globally deleted counterparts, however, these mice exhibited no liver or adipose tissue abnormalities and gained weight on HFD equal to that of controls.

Many studies have established that macrophages are central to adipose tissue homeostasis and their phenotype is distinct in obese versus lean fat. With this in mind, we deleted Asxl2 exclusively in myeloid lineage cells $\left(A s x l 2^{4 L y s M}\right)$. Whereas HFD-fed control mice increased body mass by $50 \%$ in 8 weeks, surprisingly those without Asxl2, targeted only in myeloid cells, were completely resistant to weight gain despite increased food intake and similar activity and fecal fat. Moreover, while HFD-fed control mice were insulin resistant, those with myeloid-depleted $A s x l 2$ were metabolically normal. Importantly, myeloid-specific Asxl2 deletion markedly diminished HFD-stimulated macrophage abundance in fat, and its associated inflammatory cytokine expression. Likely due to preservation of catecholamines, HFD $A s x l 2^{4 L y s M}$ BAT was protected from lipid-mediated UCP1 suppression typically attending obesity. Thus, resting energy expenditure was $45 \%$ greater in HFD-fed Asxl2 $2^{4 L y s M}$ mice than control mice. WAT of HFD-fed $A s x l 2^{4 L y s M}$ mice 
A

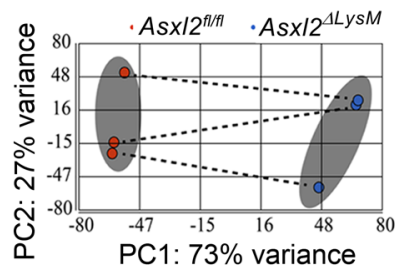

B

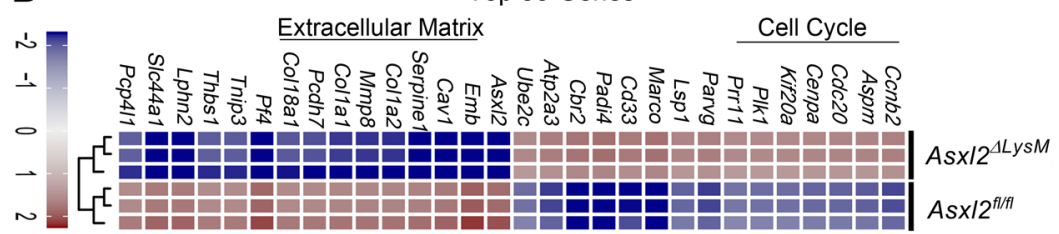

C

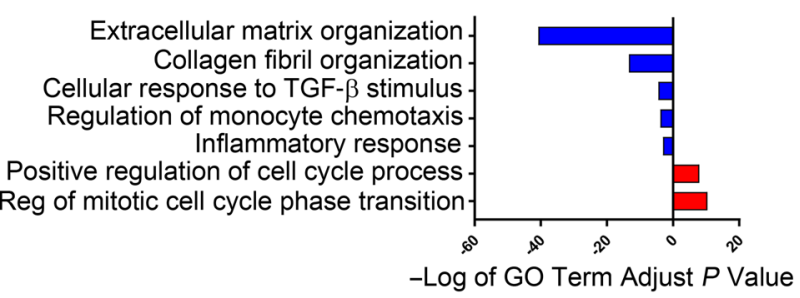

D Body Weight (g)

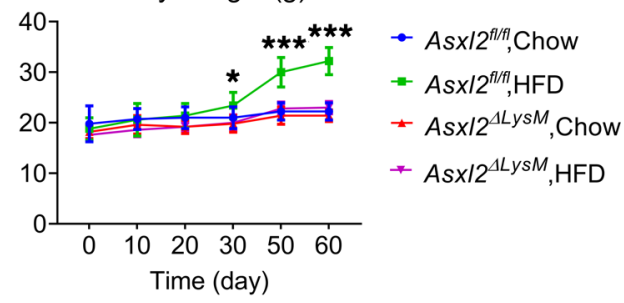

E

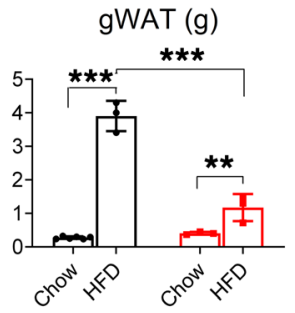

G

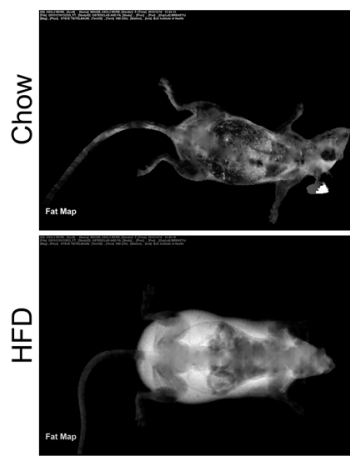

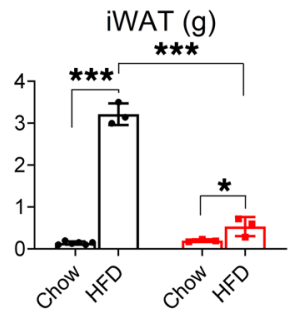

$A s \times / 2^{\triangle L y s M}$

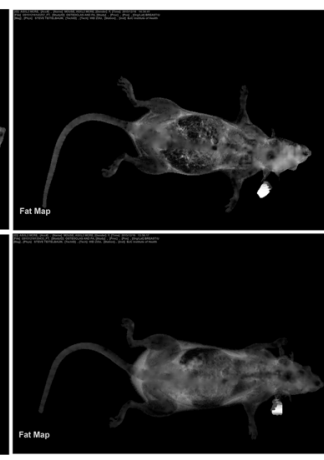

F $\quad$ Cell Area $\left(\mu \mathrm{m}^{2}\right)$

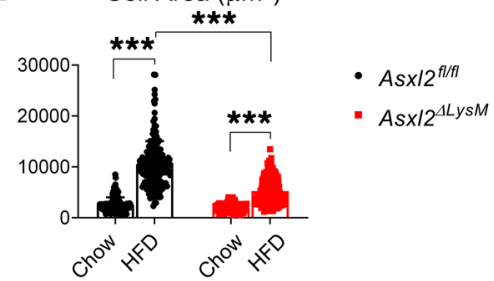

H

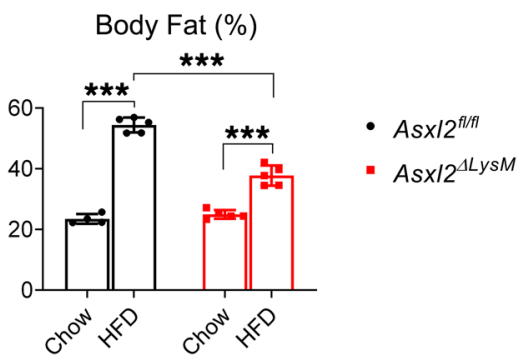

$\mathbf{K}$

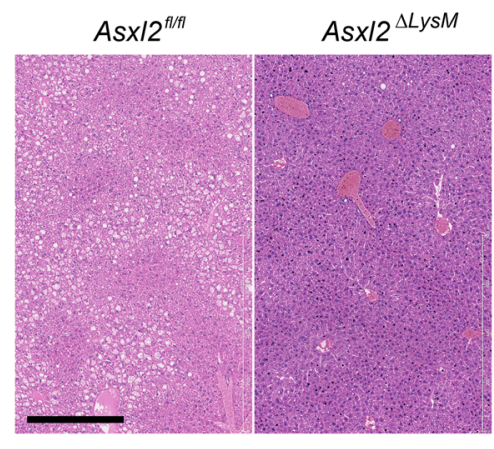

I Blood Glucose (mg/dL)
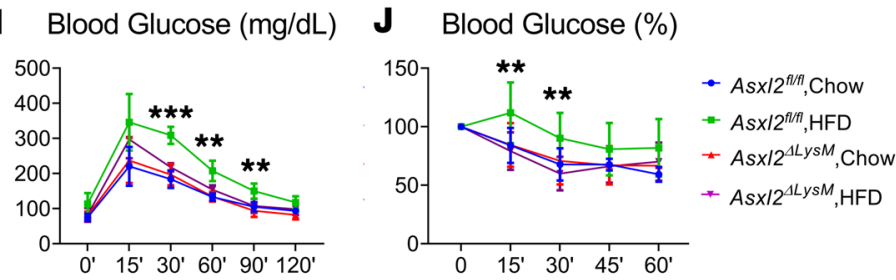

- Asx/2 $2^{\text {fiff }}$, Chow

- Asx/2 ${ }^{\mathrm{fH} / \mathrm{H}}, \mathrm{HFD}$

- As $\times 12^{\triangle L y s M}$, Chow

$\begin{array}{lllll}0 & 15^{\prime} & 30^{\prime} & 45^{\prime} & 60^{\prime}\end{array}$

Figure 1. ASXL2 regulates weight gain and metabolic homeostasis. (A-C) RNA-seq analysis of bone marrow macrophages derived from Asx/2fl/fl and As $x / 2^{2 L y s M}$ mice. (A) Principal component analysis of all differentially expressed genes. Shaded ellipses are $95 \%$ confidence intervals for each group. (B) Heatmap of the top 30 most differentially expressed genes in macrophages based on log(fold change) $>1.3$ with adjusted $P<0.001$. (C) Gene Ontology (GO) term analysis of all genes significantly downregulated (negative enrichment, blue bar) or upregulated (positive enrichment, red bar) in Asx/2 ${ }^{\text {ALysM }}$ macrophages. (D-K) Two-month-old control and $A s \times 12^{4 L y s M}$ mice were fed chow diet or HFD for 8 weeks. (D) Body weight with time. (E) Weight of gonadal WAT (gWAT) and inguinal WAT (iWAT) depots at sacrifice. (F) Size of WAT adipocytes at sacrifice. (G) DXA scans at time of sacrifice. (H) DXA-determined percentage body fat at sacrifice. (I) Glucose tolerance test performed before sacrifice. (J) Insulin tolerance test performed before sacrifice. (K) Hematoxylin and eosin-stained liver of control and $A s \times 12^{4 L y s M}$ mice after 8 weeks on HFD. Scale bar: $400 \mu \mathrm{m}$. Data are presented as mean $\pm \mathrm{SD}$. ${ }^{*} P<0.05 ;{ }^{* *} P<0.01$; ${ }^{* * *} P<0.001$; as determined by 2-way ANOVA with Holm-Sidak post hoc analysis for multiple comparisons (D-F and $\left.\mathbf{H}-\mathbf{J}\right)$. 
A Food Intake (g/day/kg)

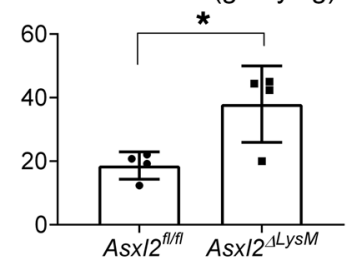

C

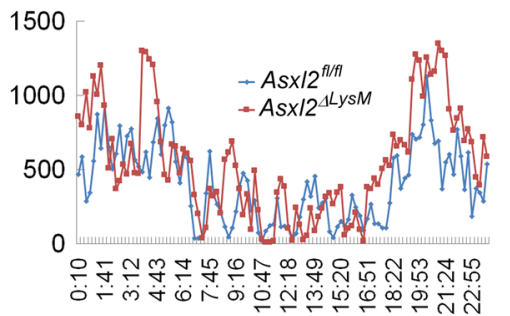

E

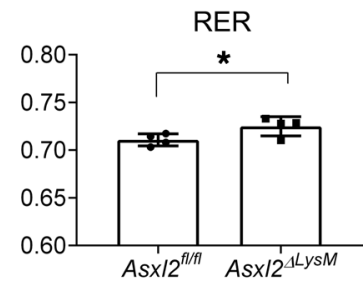

Figure 2. ASXL2 regulates energy expenditure. (A) Food intake normalized to body weight of

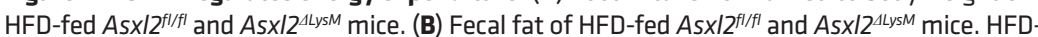
fed $A s \times 12^{f / / f l}$ and $A s \times 12^{4 L y s M}$ mice were placed in metabolic cages for 48 hours with 12-hour light/ dark cycles. (C) Activity, (D) energy expenditure, and (E) respiratory exchange rate (RER) at 48 hours. Data are presented as mean $\pm \mathrm{SD} .{ }^{*} P<0.05$, as determined by unpaired $t$ test (A and $\mathbf{E}$ ).
B

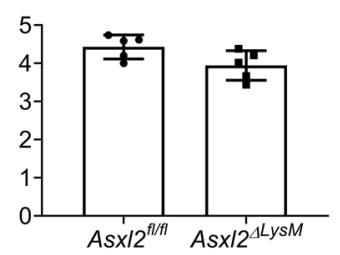

D Energy Expenditure $(\mathrm{kcal} / \mathrm{h} / \mathrm{kg})$

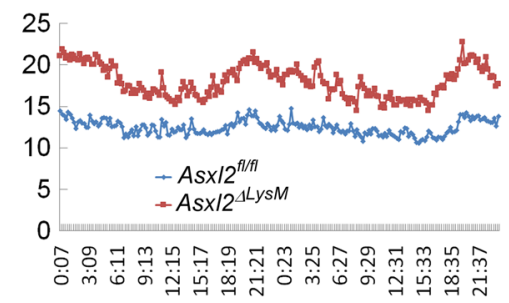

Given that adipocyte and hepatic expression of ASXL2 does not regulate its metabolic properties we turned to myeloid cells, particularly macrophages, because of their established role in the biology of obesity. Thus, we deleted Asxl2 in the myeloid lineage using LysM-Cre $\left(A s x l 2^{4 L y s M}\right)$ and characterized the transcriptomic profiles of bone marrow-derived macrophages (BMMs) by performing unbiased RNA sequencing (RNA-seq). Principal component analysis of all expressed genes (median counts per million > 1.0) in Asxl2 ${ }^{4 L y s M}$ compared with Asxl2 $2^{f / f l}$ macrophages revealed 766 significantly downregulated and 950 significantly upregulated genes ( $\mid$ fold change $\mid>1.3$, FDR $P$ value $<0.001$ ) (Figure $1 \mathrm{~A})$. Of these, the 15 most upregulated and downregulated genes are detailed in a heatmap (Figure 1B). Enrichr analysis of differentially expressed genes $(6,7)$ revealed substantial downregulation of those associated with extracellular matrix (ECM) organization, collagen formation, cytokine-cytokine interaction, and inflammatory response (Figure 1C), including genes encoding Tnfa, Mmps, and Cxcls, in Asxl2 $2^{4 L y s M}$ BMMs. In contrast, cell cycle- and mitosis-related genes were upregulated. The fact that most of the dominant downregulated genes are also implicated in modulating adipose tissue inflammation and fibrosis supports the concept that genetic deletion of Asxl2 in myeloid cells may modify obesity. Therefore, we fed normal chow or HFD to $A s x l 2^{f l f l}$ and $A s x l 2^{L L y s M}$ mice. As expected, body weights of both control and $A s x l 2^{4 L y s M}$ mice

also exhibited none of the pathological remodeling extant in their control counterparts. Finally, siRNA-mediated Asxl2 suppression in vivo, using nanoparticles (NPs) specifically targeting inflammatory macrophages, also prevented HFD-induced obesity. These experiments establish that targeting a single myeloid lineage gene can eliminate diet-induced obesity and its complications.

\section{Results}

$H F D$-fed Asxl2 ${ }^{4 L y s M}$ mice are resistant to metabolic challenge. We previously reported that global deletion of Asxl2 effects glucose and lipid metabolism (4). Unlike their WT counterparts, Asx $x 2^{-/-}$mice fed a HFD fail to gain weight, indicating a possible role for ASXL2 in metabolically active tissue. Given these observations and the fact that ASXL2 controls PPAR $\gamma$-dependent gene expression (4), which is required for adipogenesis, we hypothesized that global Asxl2 deficiency prevents adipogenesis by disrupting PPAR $\gamma$ in fat. To test this hypothesis, we mated $A s x l 2^{f / f l}$ mice with those expressing adiponectin-Cre to selectively delete Asxl2 in adipocytes (Asxl2 ${ }^{\text {AAdipoq }}$ (5). Contrary to our hypothesis, Asxl2 deficiency in adipose tissue did not affect body weight at steady state nor in response to HFD (Supplemental Figure 1A; supplemental material available online with this article; https://doi.org/10.1172/JCI128687DS1). The same holds regarding liver-selective $A s x l 2$ deletion using albumin-Cre $\left(A s x l 2^{4 A l b}\right)$ (Supplemental Figure 1B). Additionally, glucose tolerance of HFD-fed $A s x l 2^{\triangle A d i p o q}$ and $A s x l 2^{\triangle A l b}$ mice mirrored their Cre-negative counterparts (Supplemental Figure 1, C and D) were similar on chow diet, while those of HFD-fed control mice $\left(A s x l 2^{f / f l}\right)$ increased approximately $50 \%$ after 8 weeks (Figure 1D). Surprisingly, those lacking Asxl2 exclusively in myeloid lineage cells were completely impervious to diet-induced weight gain, mirroring their chow-fed counterparts. Consistent with these observations, inguinal WAT (iWAT) and gonadal WAT (gWAT) weights, as well as adipocyte size, were substantially less in $A s x l 2^{4 L y s M}$ mice compared with $A s x l 2^{f / f l}$ controls on an HFD (Figure 1, E and F). Dual-energy x-ray absorptiometry (DXA) scans showed markedly increased visceral and subcutaneous adiposity in HFD-fed $A s x l 2^{f l / f l}$ controls but only a modest gain in analogous $A s x l 2^{4 L y s M}$ mice (Figure $1, \mathrm{G}$ and $\mathrm{H}$ ). Asxl2 $2^{4 L y s M}$ mice were also protected from HFD-induced glucose and insulin intolerance (Figure 1, I and J) and $A s x l 2^{4 L y s M}$ mice, but not $A s x l 2^{f / f l}$ mice, did not develop diet-induced hepatic steatosis (Figure $1 \mathrm{~K}$ ). Thus, diet-induced metabolic syndrome is prevented by ASXL2 inactivation in myeloid lineage cells.

Obesity resistance of myeloid-specific Asxl2 deletion reflects relatively increased energy expenditure. Despite failure to gain weight, food intake of HFD-fed Asxl2 $2^{4 L y s M}$ mice, normalized to body weight, surpassed that of their obese control counterparts, while fecal fat content was the same (Figure 2, A and B). These observations raised the prospect that resistance to diet-induced weight gain of $A s x l 2^{4 L y s M}$ mice reflects relatively enhanced energy utilization. To address this possibility, control and $A s x l 2^{4 L y s M}$ mice were placed in metabolic cages for 48 hours, 8 weeks after initiation 
A

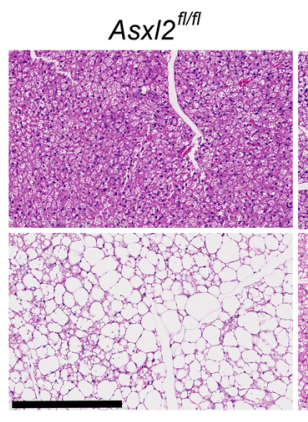

D

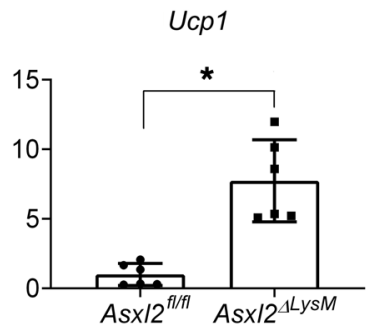

B

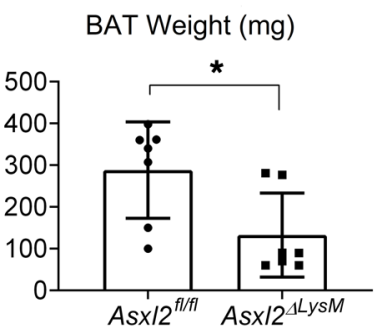

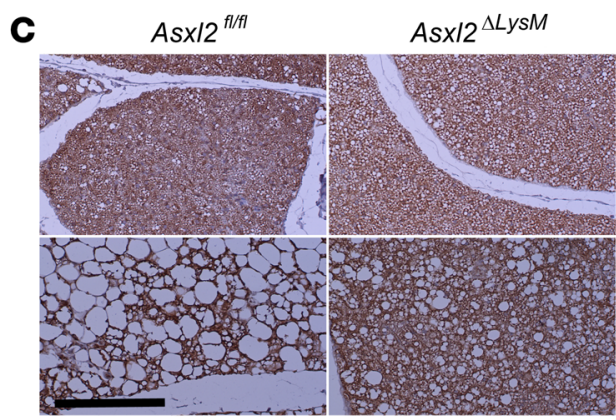

F

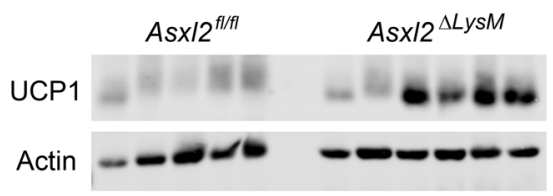

H

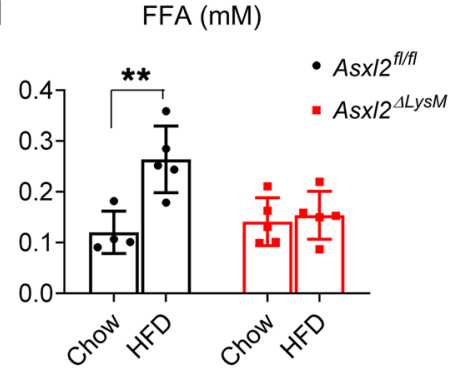

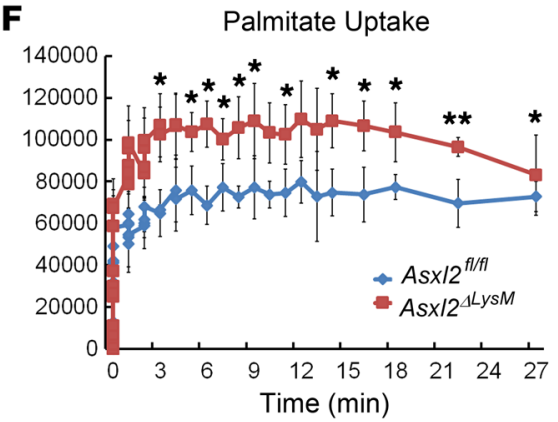

I Triglycerides (mg/dL)

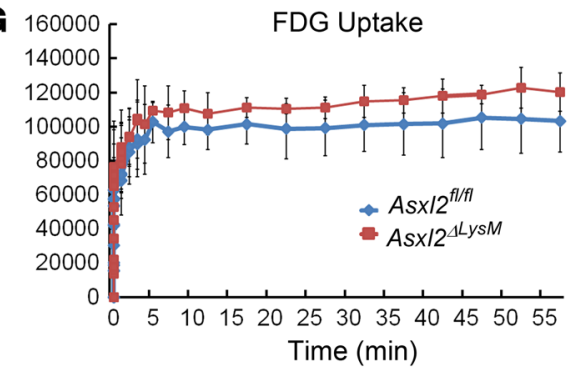

J

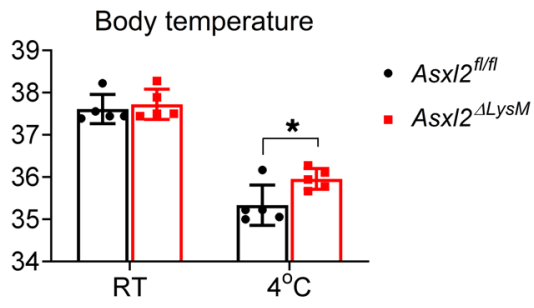

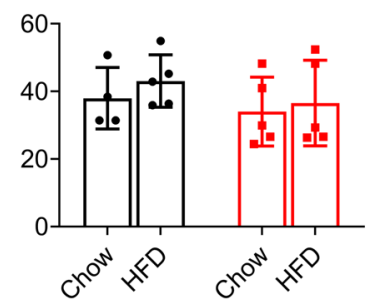

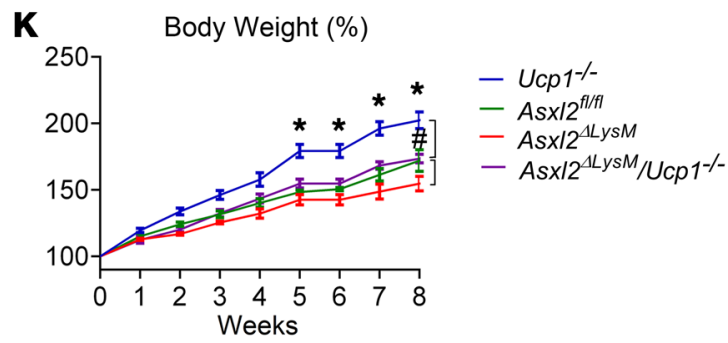

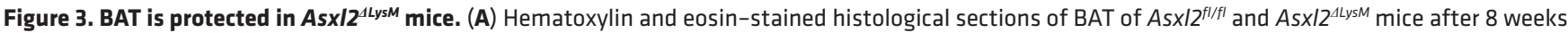
on chow diet or HFD. Scale bar: $300 \mu \mathrm{m}$. (B) BAT weight of HFD-fed Asx/2f/ff and Asx/2 $2^{1 L y s M}$ mice. (C) UCP1-immunostained histological sections of BAT of

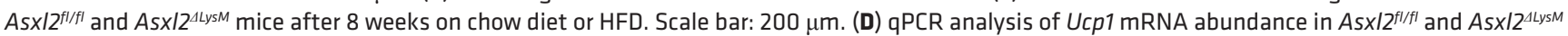
mice after 8 weeks on HFD. (E) Immunoblot of UCP1 abundance in AsxI2 $2^{f l f l}$ and $A s \times 12^{4 L y s M}$ mice after 8 weeks on HFD. (F and G) PET scan determination of (F) palmitate and (G) fludeoxyglucose metabolism in BAT of $A s \times 12^{f l / f l}$ and $A s \times 12^{4 L y s M}$ mice after 8 weeks on HFD. (F) Serum free fatty acids (H) and triglycerides (I) of $A s \times 12^{f / / f l}$ and $A s \times 12^{\Lambda L y s M}$ mice after 8 weeks on chow diet or HFD. (J) Core body temperature of $A s \times / 2^{f / / f l}$ and $A s \times 12^{4 L y s M}$ mice maintained at

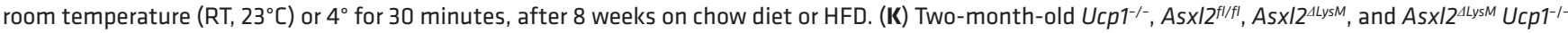
mice were fed HFD for 8 weeks at neutral thermal temperature $\left(30^{\circ} \mathrm{C}\right)$; body weight change was measured with time. ${ }^{*} P<0.05$ for comparisons between

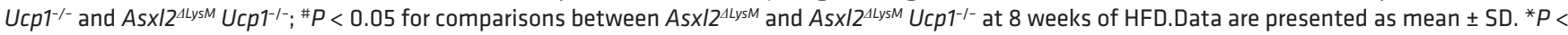
0.05 ; ${ }^{*} \mathrm{P}<0.01$; as determined by unpaired $t$ test (B and $\mathbf{D}$ ) or 2-way ANOVA with Holm-Sidak post hoc analysis for multiple comparisons (F, $\mathbf{H}, \mathbf{J}$, and $\mathbf{K}$ ).

of HFD. Despite equivalent physical activity (Figure 2C), energy expenditure, normalized to body weight, was approximately $45 \%$ greater, through light and dark cycles, in $A s x \mid 2^{4 L y s M}$ mice than similarly fed controls (Figure 2D). The respiratory exchange ratio (RER) was also greater in HFD Asxl2 $2^{4 L y^{s} \mathrm{sM}}$ mice (Figure 2E). Thus, the obesity resistance of $A s x 2^{24 y s s M}$ mice reflects a relative increase in energy utilization.
$B A T$ is protected in Asxl2 $2^{4 L y s M}$ mice. The energy expenditure of HFD-fed Asxl2 ${ }^{4 L y s M}$ mice raised the possibility that it reflects uncoupling of ATP and respiration, as induced by UCP1. Thus, we explored beiging of iWAT. Like their control counterparts, chowfed $A s x l 2^{24 y_{5} s}$ mice contained numerous foci of characteristic, UCP1-expressing beige fat foci that were absent in HFD-fed control animals (Supplemental Figure 2A). Despite their normal met- 
A Tyrosine Hydroxylase

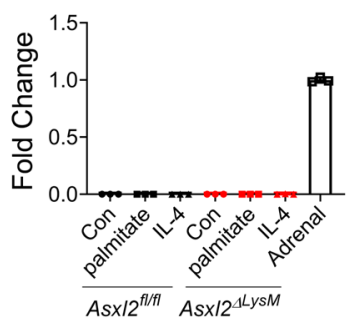

B

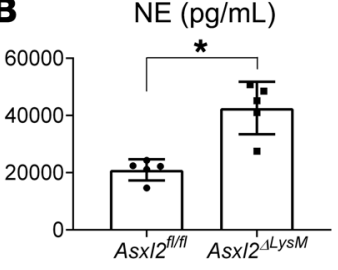

C Glycerol Released $(\mathrm{mM} / \mathrm{g})$

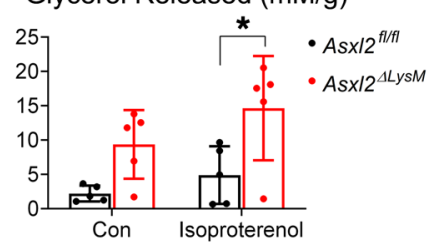

D

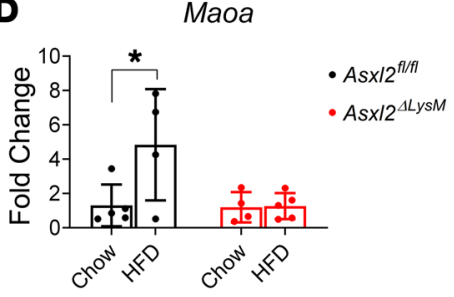

abolic phenotype, iWAT of HFD-fed $A s x l 2^{L y s M}$ mice surprisingly mirrored that of their control counterparts, as it was also devoid of UCP1-producing adipocytes. Thus, the robust energy expenditure induced by myeloid-specific Asxl2 deletion likely does not represent iWAT beiging.

Similarly to WAT, the appearance of brown adipose tissue (BAT) of chow-fed $A s x l 2^{4 L y s M}$ mice was indistinguishable from their control counterparts (Figure 3A). As previously noted, HFD induces whitening of BAT in $A s x l 2^{f / f l}$ mice in that adipocytes contain large lipid droplets reminiscent of those in WAT $(8,9)$. Although BAT of HFD-fed $A s x l 2^{4 L y s M}$ mice exhibited some evidence of whitening compared with that of chow-fed animals, it was minimal relative to that of HFD-fed $A s x l 2^{f / f l}$ mice. Consequent to avoidance of diet-induced lipid accumulation, the weight of HFD Asxl2 ${ }^{4 L y s M}$ BAT was lower than that of control (Figure 3B). In keeping with evidence that prevention of HFD-induced whitening preserves BAT activity (10), UCP1 expression determined by immunostaining, quantitative real-time PCR (qPCR), and immunoblot, was relatively enhanced in HFD Asx $l 2^{4 L y s M}$ mice (Figure 3, C-E, and Supplemental Figure 2B). Furthermore, PET imaging documented greater palmitate metabolism by HFD $A s x l 2^{4 L y s M}$ than $A s x l 2^{f l / f l} \mathrm{BAT}$, in vivo (Figure $3 F$ ). Although fludeoxyglucose metabolism by HFD As $x l 2^{4 L y s M}$ BAT trended higher relative to that of $A s x l 2^{f l / f l}$ counterparts, the difference was not significant (Figure 3G). Circulating free fatty acids (FFAs) were increased in HFD control mice but not those with myeloid Asxl2 deletion, likely reflecting maintenance of their robust metabolism in BAT (Figure $3 \mathrm{H}$ and ref. 11). In keeping with previous data, serum triglycerides were unaltered in each cohort of mice regardless of diet (Figure 3I).

As in mice, human fat mass and BAT activity are negatively correlated, whereas BAT activity and energy expenditure are positively related (12). Hence, the obesity resistance of $A s x l 2^{4 L y s M}$ mice may represent their preserved UCP1-mediated energy expenditure. In keeping with the hypothesis that their failure to gain weight represents preservation of UCP1-mediated ATP uncoupled respiration, core body temperature of HFD $A s x l 2^{4 L y s M}$ mice, maintained at $4^{\circ} \mathrm{C}$, was significantly higher than that of control (Figure 3J). Asxl2 ${ }^{4 L y s M}$ mice on an $\mathrm{HFD}$, housed at $30^{\circ} \mathrm{C}$, also had lower body

Figure 4. Catecholamines are relatively increased in Asx/2 ${ }^{4 L y s M}$ BAT. (A) Tyrosine hydroxylase mRNA expression by naive and IL-4- or palmitate-treated $A s \times 12^{f / f l}$ and $A s \times / 2^{\Lambda L y s M} B M M s$. Adrenal tissue served as positive control. (B) Norepinephrine (NE) content of BAT of HFD-fed control and Asx/2 $2^{4 L y s M}$ mice. (C) Glycerol released from BAT explants derived from HFD-fed control and Asx/2 ${ }^{4 L \nu s M}$ mice. (D) Maoa mRNA abundance in BAT stromal vascular fraction of chow- or HFD-fed control and Asx/2 ${ }^{4 L 5 S M}$ mice. Data are presented as mean $\pm \mathrm{SD} .{ }^{*} P<0.05$, as determined by unpaired $t$ test (B) or 2-way ANOVA with Holm-Sidak's post hoc analysis for multiple comparisons (C and $\mathbf{D}$ ).

weight than $A s x l 2^{f l / f l}$ controls (Figure $3 \mathrm{~K}$ ). Indicating that UCP1 contributes to obesity resistance of the myeloid-mutant mice at thermoneutrality, the percentage increase in body weight of HFDfed $A s x l 2^{4 L y s M}$ mice, globally lacking $U c p 1$ (Asxl2 $\left.{ }^{4 L y s M} U c p 1^{-/-}\right)$, mirrored that of $A s x l 2^{f l / f l}$ and was significantly greater than $A s x l 2^{4 L y s M}$ (Figure 3K). Thus, UCP1 contributes to the obesity resistance of Asxl2 ${ }^{\triangle L y s M}$ mice at room temperature and thermoneutrality (13).

Catecholamines are increased in ASXL $2^{4 L y s M} B A T$. Macrophages are proposed to influence weight gain by altering uncoupled respiration. A common hypothesis holds that alternatively activated myeloid cells express catecholamines that promote UCP1 expression by beige adipocytes, thereby enhancing energy expenditure (14). Recent data, however, challenge this postulate and it has been concluded that alternatively activated macrophages do not express catecholamines (15). Consistent with this conclusion, naive and palmitate- or IL-4-treated $A s x l 2^{f / f l}$ and $A s x l 2^{\perp L y s M}$ BMMs expressed little tyrosine hydroxylase mRNA (Th), whose product catalyzes a rate-limiting step in catecholamine synthesis (Figure $4 \mathrm{~A})$. It is likely, therefore, that macrophages do not produce catecholamines that contribute to the obesity resistance of $A s x l 2^{4 L y s M}$ mice. Despite a paucity of tyrosine hydroxylase expression in macrophages, norepinephrine was unexpectedly approximately twice as abundant in HFD-fed $A s x 2^{4 L y s}$ BAT as control (Figure 4B). Because processing of triglycerides to FFAs and glycerol is regulated by norepinephrine, we measured lipolysis in BAT explants derived from HFD-fed control and Asx $x 2^{4 L y s M}$ mice. Corroborating palmitate uptake and confirming the functional significance of catecholamine abundance, lipolysis in $A s x l 2^{4 L y s M}$ BAT explants was increased, both in the basal state and when stimulated with isoproterenol (Figure 4C). Thus, despite their likely failure to produce norepinephrine, Asxl2-deficient macrophages may regulate BAT metabolism by affecting catecholamine signaling.

Although a subset of macrophages associated with sympathetic neurons do not express catecholamines, they incorporate and degrade norepinephrine, thus preventing its accumulation in BAT $(16,17)$. These sympathetic neuron-associated macrophages reduce catecholamines in BAT via expression of solute carrier member 2 (Slc6a2) norepinephrine transporter and/or monoamine oxidase A (Maoa), an amine-degrading mitochondrial enzyme. Indicating that impaired catecholamine degradation likely contributes to the preservation of BAT in HFD-fed Asxl2 ${ }^{4 L y s M}$ mice, Maoa in the stromal vascular fraction (SVF) of BAT was diminished relative to their Asxl2 $2^{f / f l}$ counterparts and mirrored that of chow-fed mice (Figure 4D).

Absence of Asxl2 in myeloid lineage cells prevents accumulation of macrophages in WAT and BAT in response to HFD. Adipose tissues contain diverse myeloid cell types, including abundant eosinophils 
Gonadal WAT

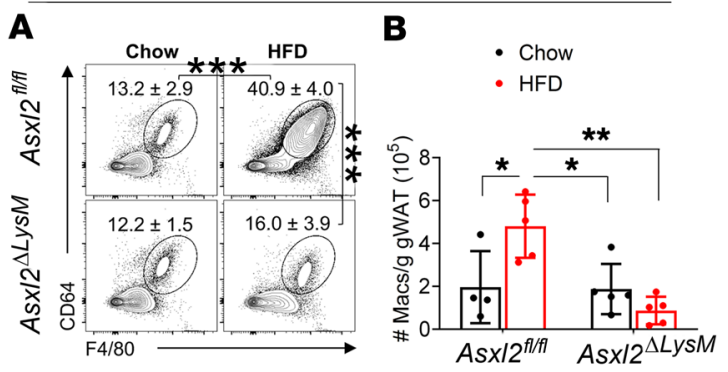

BAT
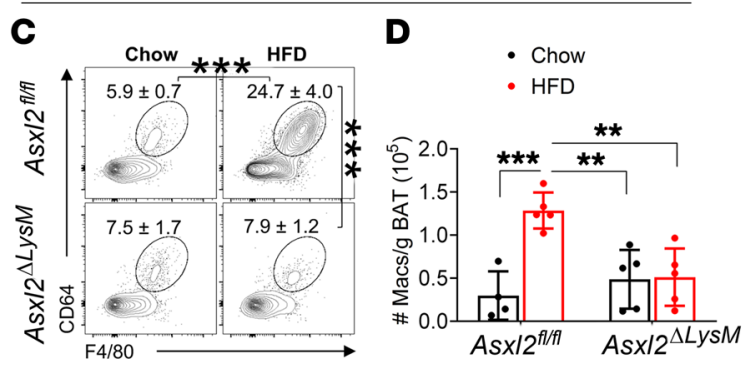

E

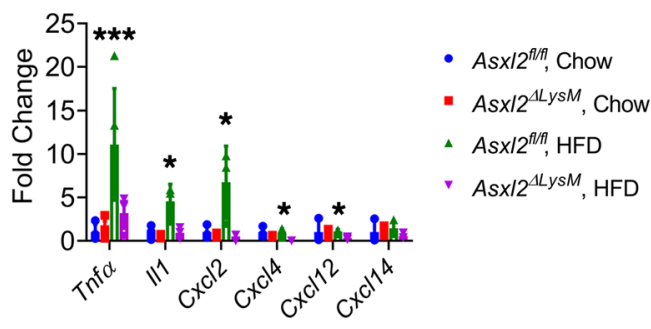

F
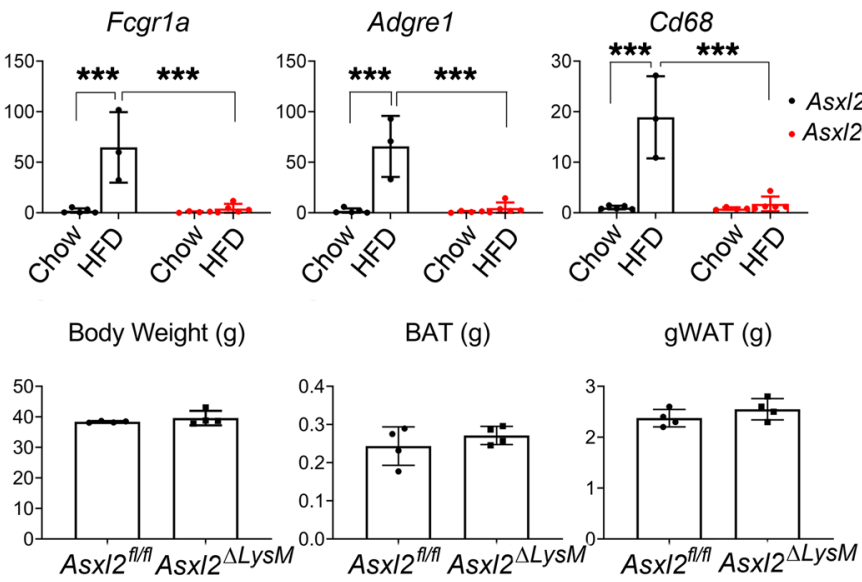

G

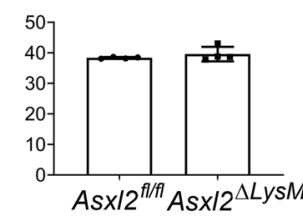

BAT (g)

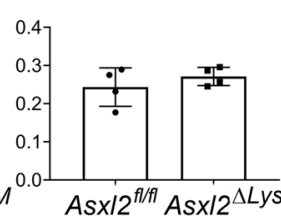

H
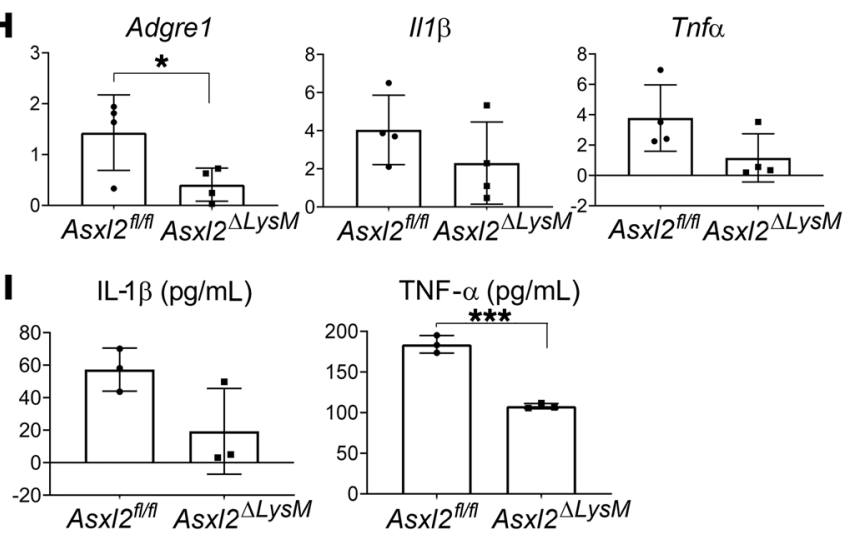

J

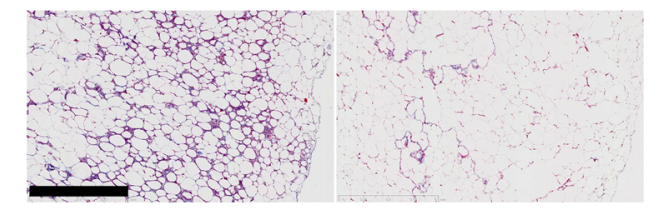

$\mathbf{K}$

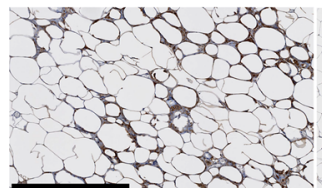

$\mathbf{L}$

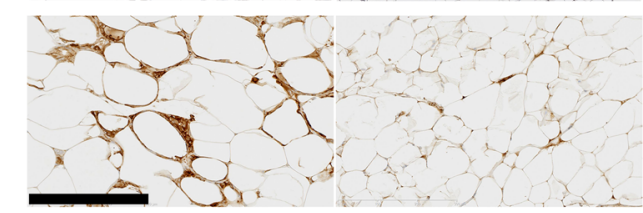

As $\times 12^{f / f l}$
M

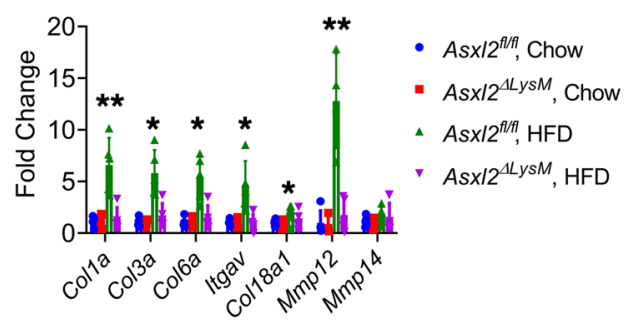

Figure 5. ASXL2 expression in myeloid cells is required for macrophage accumulation in WAT and BAT in obesity. As $x / 2^{f / f f}$ and $A s \times / 2^{4 L y s M}$ mice were fed either a chow diet or HFD. (A) Frequencies and (B) numbers of F4/80+CD64+ macrophages in gonadal WAT: pregated on singlet, live, CD45+ cells. (C) Frequencies and (D) numbers of F4/80+CD64+ macrophages in BAT: pregated on singlet, live, $C D 45^{+}$cells. (E) Inflammatory cytokine and chemokine mRNA expression in stromal vascular fraction of BAT of $A s \times 12^{f / f f l}$ or $A s \times 12^{1 L \nu s M}$ mice after 8 weeks fed with chow diet or HFD. (F) Macrophage marker mRNA expression in stromal vascular fraction of BAT of $A s \times 12^{f / f f l}$ or $A S X L 2^{4 L y s M}$ mice after 8 weeks fed with chow diet or HFD. (C) Body weight and brown or gonadal fat pad weight of $A s \times 12^{f l / f l}$ and $A s \times 12^{4 L y s M}$ mice after 4 weeks of HFD. (H) Adgre1 (F4/80) and inflammatory cytokine mRNA expression in stromal vascular fraction of BAT of $A s \times 2^{f / / f l}$ or $A s \times 12^{1 L y s M}$ mice after 4 weeks on HFD. (I) IL-1 $\beta$ and TNF- $\alpha$ secretion by bone marrow-derived macrophages of $A s \times 12^{f / /}$ ${ }^{f l}$ or $A s \times 12^{4 L 4 s M}$ mice stimulated with $100 \mathrm{ng} / \mathrm{mL}$ LPS for 3 hours, followed by $15 \mu \mathrm{M}$ nigericin for 1 hour. (J-L) Histological sections of WAT of HFD-fed control or Asx/2 ${ }^{\text {LLys } M}$ mice stained to identify (J) fibrosis (hematoxylin and eosin), (K) hypoxic adipocytes, and (L) crown-like structures (F4/80). Scale bars: 1 mm (J), $400 \mu \mathrm{m}(\mathbf{K})$, and $200 \mu \mathrm{m}$ (L). (M) ECM gene mRNA expression in gonadal WAT stromal vascular fraction of chow- or HFD-fed Asx/2fl/fl or Asx/2 4 LysM mice. Data are presented as mean $\pm \mathrm{SD}$. ${ }^{*} P<0.05$; ${ }^{* *} P<0.01$; ${ }^{* *} P<0.001$; as determined by unpaired $t$ test (H and $\mathbf{I}$ ) or 2-way ANOVA with Holm-Sidak post hoc analysis for multiple comparisons (B, D-F, and $\mathbf{M})$. 

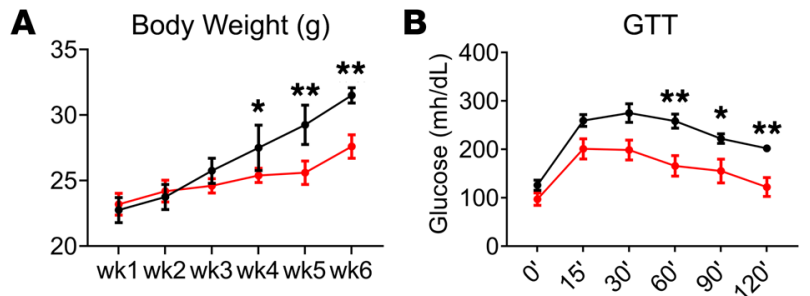

C

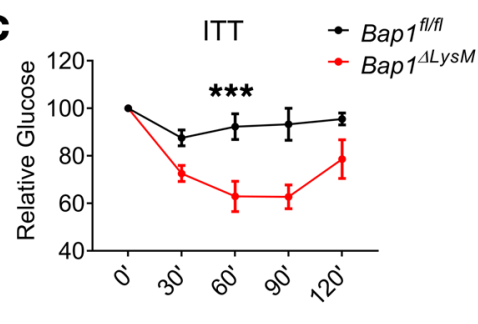

Merge
D

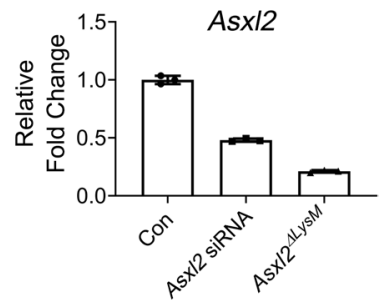

E

$\mathrm{F} 4 / 80$

siRNA Nanoparticle

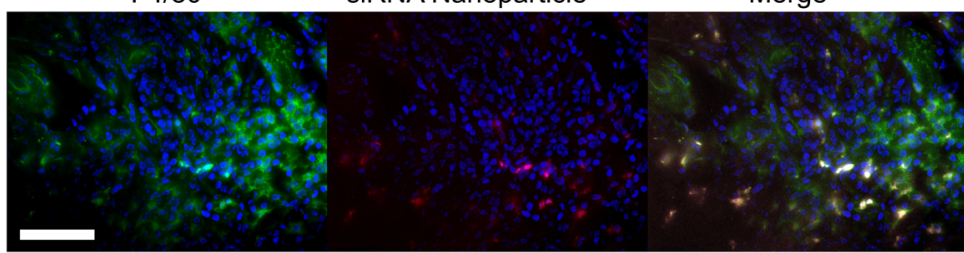

$\mathbf{F}$

$\mathrm{F} 4 / 80$

siRNA Nanoparticle

Merge

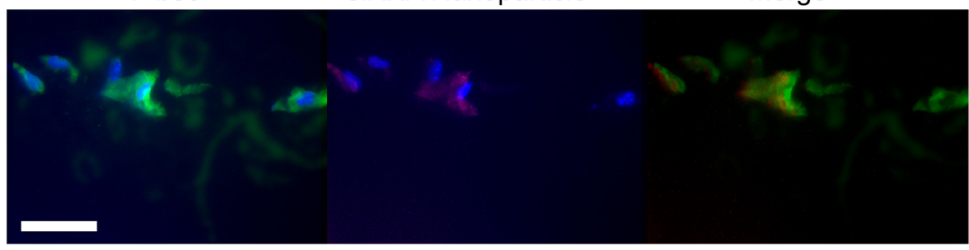

\section{G}

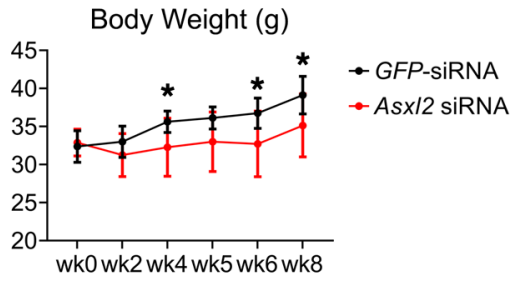

Figure 6. siRNA-mediated Asx/2 suppression in macrophages prevents diet-induced obesity. (A) Body weight, (B) glucose tolerance test, and (C) insulin tolerance test of Bap $7^{f / f f}$ and Bap ${ }^{1 L \text { Lys }}$ mice fed HFD for 6 weeks. (D) WT BMMs were incubated with GFP-siRNA- or Asx/2-siRNA-associated nanoparticles. Asx/2 mRNA was measured by qPCR and compared to that of AsxI2 ${ }^{4 L y s M}$ BMMs. Colocalization of macrophages (F4/80) and Asx/2-siRNA-associated nanoparticles in (E) spleen and (F) gonadal WAT of HFD-fed WT mice. Scale bars: $100 \mu \mathrm{m}(\mathbf{E})$ and $30 \mu \mathrm{m}$ (F). (C) Body weight of WT HFD-fed mice administered GFP-siRNA- or Asx/2-siRNA-associated nanoparticles. Data are presented as mean \pm SD. ${ }^{*} P<0.05 ;{ }^{* *} P<0.01$; ${ }^{* *} P<0.001$; as determined by 1-way ANOVA with Holm-Sidak post hoc analysis for multiple comparisons (A-C and $\mathbf{G}$ ).

and macrophages. In the lean state, eosinophils produce IL-4 to sustain an antiinflammatory program in WAT macrophages (18). In obesity, however, eosinophils in WAT are reduced and macrophages upregulate expression of proinflammatory cytokines such as TNF- $\alpha$ that impair glucose homeostasis $(19,20)$. We therefore asked whether ASXL2 expression in myeloid lineage cells determines eosinophil or macrophage responses to HFD in WAT and BAT.

We identified $\mathrm{CD} 45^{+}$Siglec $\mathrm{F}^{+} \mathrm{SSC}^{\text {hi }}$ eosinophils and $\mathrm{CD} 45^{+} \mathrm{F} 4 /$ $80^{+} \mathrm{CD} 64^{+}$macrophages in WAT and compared their abundance in $A s x l 2^{f l / f l}$ and $A s x l 2^{A L y s M}$ mice (Supplemental Figure 3). Eosinophils were decreased in WAT of HFD-fed mice irrespective of genotype, suggesting that ASXL2 does not determine eosinophil responses to diet-induced obesity (Supplemental Figure 4). In contrast, WAT macrophages were increased in frequency and number following an HFD in $A s x l 2^{f l / f l}$ mice, but not in Asxl2 ${ }^{4 L y s M}$ mice (Figure 5, A and B). A similar pattern occurred regarding macrophage frequencies and numbers in BAT (Figure 5, C and D). Consistent with this observation and confirming previous RNA-seq data, HFD induced expression of inflammatory cytokine mRNAs, including $I l 1 b$ and Tnfa, in both BAT (Figure 5E) and WAT (Supplemental Figure 5) SVF of HFD-fed Asxl2 $2^{f / f l}$ but not Asxl2 $2^{4 L y s M}$ mice. Additionally, macrophages expressing Adgre1 (F4/80), Cd68, and Fcgr1a (CD64) were decreased in $A s x l 2^{4 L y s M}$ BAT SVF (Figure 5F). To determine if the decreased macrophage infiltration in BAT is a consequence of myeloid deletion of $A s x l 2$ or of obesity resistance, we fed mice an HFD for 4 weeks, at which time body weight of WT mice had not yet increased (Figure $5 G$ ). Confirming that the relative paucity of macrophages in HFD-fed $A s x l 2^{4 L y s M}$ mice is not due to their failure to gain weight, expression of the macrophage markers Adgre1 (F4/80), Tnfa, and Illb was significantly lower in BAT SVF of 4-week HFD-fed $A s x l 2^{4 L y s M}$ mice than similarly fed, nonobese $A s x l 2^{f l / f l}$ controls (Figure $5 \mathrm{H}$ ).

Induction of the NLRP3 inflammasome enhances MAOA abundance (16). The paucity of IL-1 $\beta$ mRNA, expressed by Asxl2 $2^{4 L y s M}$ relative to $A s x l 2^{f / f l}$ macrophages, therefore raised the possibility that preservation of BAT catecholamines reflects suppressed inflammasome activity. Expression of Nlrp3 by Asxl2 ${ }^{4 L y s M}$ and $A s x l 2^{f / f l}$ BMMs, in response to LPS or TNF- $\alpha$ was, however, similar, suggesting that NLRP3 is not regulated by ASXL2 (Supplemental Figure 6A). Additionally, LPS- and nigericin-induced inflammasome activation, reflected by cellular foci upon binding of the FLICA probe to active caspase-1 (21), yielded no difference between $A s x l 2^{4 L y s M}$ and $A s x l 2^{f l / f l}$ macrophages (Supplemental Figure 6B). Interestingly, LPS-stimulated secretion of IL- $1 \beta$ by $A s x$ $l 2^{4 L y s M}$ BMMs was decreased, suggesting that ASXL2 inactivation dampens pathways involved in the secretion, but not maturation, of IL-1 $\beta$ (Figure 5I). Likewise, TNF- $\alpha$ expression by mutant cells was decreased (Figure 5I). It is therefore likely that absence of ASXL2 in macrophages preserves catecholamines by their limited infiltration into BAT and diminished expression and secretion of proinflammatory cytokines. Supporting this conclusion, no differences in Maoa expression were observed in $A s x l 2^{4 L y s M}$ and control BMMs (Supplemental Figure 6C). 
Obesity is characterized by destructive changes in WAT, including hypovascularity-induced hypoxia leading to adipocyte death $(19,22)$ and eventuating in peri-adipocyte fibrosis that compromises lipid uptake and, therefore, metabolic function. The fibrosis of obese WAT enhances the inflammatory state of pre-adipocytes and macrophages, thus invoking a cycle of ECM production, inflammation, and pathological adipose tissue remodeling (23). Like their gross phenotype, the microscopic appearance of WAT of chow-fed $A s x l 2^{4 L y s M}$ mice was indistinguishable from their control counterparts (not shown). As expected, WAT of $A s x l 2^{f l / f l}$ mice, maintained on HFD, contained abundant fibrosis, a plethora of hypoxic cells, and numerous crown-like structures in which macrophages phagocytose lipid droplets (Figure 5, J-L). In contrast, adipocytes of HFD-fed Asxl2 $2^{4 L y s M}$ mice exhibited no evidence of fibrosis, crown-like structures, or hypoxic cells. In keeping with these morphological observations, HFD promoted expression of selected collagen synthesis genes in WAT SVF in $A s x l 2^{f / f l}$ mice but not $A s x l 2^{4 L y s M}$ counterparts (Figure $5 \mathrm{M}$ ). These observations indicate that ASXL2 is required for macrophage accumulation and inflammation in WAT and BAT in the context of diet-induced obesity and suggest that ASXL2 expression in macrophages may contribute to pathological remodeling of fat.

Myeloid-specific BAP1 deletion arrests HFD-induced weight gain. BAP1 is a tumor suppressor and deubiquitinase that complexes with and stabilizes ASXL2 (24). This relationship raised the possibility that, like ASXL2, absence of BAP1 in myeloid lineage cells impacts HFD-induced weight gain. Thus, mirroring our approach to ASXL2, we mated Bap1 $1^{f / f l}$ and LysM-Cre mice. Similarly to Asxl2 ${ }^{4 L y s M}$ mice, those lacking Bap1 exclusively in myeloid lineage cells failed to gain weight on an HFD and maintain glucose and insulin sensitivity (Figure 6, A-C). Like Asxl2 ${ }^{4 L y s M}$ mice, HFD-fed Bap1 $1^{4 L y s M}$ mice had diminished Maoa and arrested lipolysis in BAT (Supplemental Figure 7). Given its essential role in stabilizing ASXL2, the obesity-preventing properties of myeloid-specific deletion of Bap1 is in keeping with the similar phenotype of $A s x l 2^{4 L y s M}$ mice.

siRNA-mediated ASXL2 suppression in macrophages prevents diet-induced obesity. Our data raise the possibility that therapeutic suppression of ASXL2 expression in macrophages may obviate diet-induced obesity. To explore this prospect, we turned to Asxl2 siRNA formulated with a biocompatible cationic amphipathic peptide (p5RHH) to create 55-nm NPs that are phagocytosed by macrophages, followed by endosomolysis and siRNA release to engage the RISC complex (25-27). The clinical potential of this strategy is underscored by the fact that NP-mediated ablation of specific subsets of myeloid lineage cells positively affects conditions such as stroke and sepsis (28) and the same macrophage-targeting NPs, used here, combined with NF-кB p65 siRNA, prevent inflammatory and osteoarthritis $(29,30)$. We therefore asked if a similar strategy to suppress macrophage ASXL2 expression would arrest diet-induced obesity. To this end, we used a Cy5.5labeled p5RHH-Asxl2 siRNA, designed and synthesized by SigmaAldrich, multiplexed in unitary peptidic NPs, which suppressed Asxl 2 mRNA expression in isolated BMMs by approximately $50 \%$ (Figure 6D). We administered $0.5 \mathrm{mg} / \mathrm{kg}$ to mice, twice per week while maintaining them on an HFD for 8 weeks.
Distribution of the p5RHH-siRNA complex in mice with collagen-induced arthritis appears limited to inflamed joints and kidneys. In contrast to the relatively restricted localization in inflammatory arthritis and perhaps reflecting the systemic inflammation accompanying obesity (22), Asxl2-siRNA-associated NPs were distributed in kidneys, spleen, bone, muscle, lung, and subcutaneous and visceral fat depots (Supplemental Figure 8). Confirming that they target macrophages, Asxl2-siRNA-associated NPs localized exclusively in cells expressing F4/80 in spleen and targeted the same cells in gWAT of HFD-fed WT mice (Figure 6, E and F). Administration of this NP complex to HFD-fed WT mice completely prevented weight gain relative to those receiving GFP-siRNA-associated NPs (Figure 6G). Thus, pharmacological arrest of Asxl2 expression in macrophages has antiobesity properties. Furthermore, prevention of diet-induced weight gain by macrophage-specific siRNA-associated NPs fortifies the myeloid specificity of LysM-mediated Asxl2 ablation.

\section{Discussion}

Resistance of $A s x l 2^{4 L y s M}$ mice to diet-induced weight gain suggests obesity can be positively affected by manipulation of myeloid lineage cells. Furthermore, evidence that abundant fat intake may prolong longevity in humans and mice buttresses the potential importance of possibly limiting obesity in the face of $\operatorname{HFD}(31,32)$.

This study was initiated with the expectation that absence of the gene in classical metabolism-regulating tissues, namely liver or fat, mediates the obesity resistance of global Asxl2 deletion. Although that proved not to be the case, mice bearing myeloid-specific deletion of the ETP gene mirrored their globally deficient counterparts in that they failed to gain HFD-induced weight. Importantly, unlike those with global deletion, insulin and glucose homeostases of Asx$l 2^{4 L y s M}$ mice on HFD remain preserved. Fortifying the concept that myeloid-expressed ASXL2 regulates diet-induced obesity, similar conditional deletion of Bap1, which stabilizes the ETP protein and enables its epigenetic properties, also prevents HFD-induced weight gain and its metabolic consequences $(24,33)$.

In the face of enhanced food consumption, unaltered activity, and normal intestinal absorption of HFD-fed Asxl2 ${ }^{4 L y s M}$ mice, it is likely their obesity resistance reflects enhanced basal energy expenditure relative to similarly fed $A s x l 2^{f / f l}$ controls. Furthermore, the relative increase in BAT metabolic activity, as determined by fatty acid incorporation and UCP1 abundance, suggests that protection of BAT from the effects of obesity by myeloid-specific deletion of Asxl2 is substantially responsible for increased energy expenditure of the mutant mice. On the other hand, Asxl2 $2^{4 L y s M}$ macrophage-mediated prevention of steatosis, which reduces hepatocyte $\beta$-oxidation, may also contribute to the relatively robust energy expenditure of the conditionally deleted mice (34).

Morphologically, the most impressive difference between HFD control and $A s x l 2^{4 L y s M}$ BAT is accumulation of large lipid droplets in the former. This whitening diminishes BAT efficacy. Given their reduced basal energy expenditure, it is possible that lipid droplet accumulation in BAT may contribute to the predisposition of previously obese individuals to regain weight. Although prevention of whitening of $A s x l 2^{4 L y s M}$ BAT is likely central to maintaining energy expenditure, the means by which lipid accumulation in this tissue is prevented in our mutant is enigmatic. 
In normal HFD obesity, hypoxia-induced necrotic adipocytes recruit and activate macrophages which, in turn, induce a proinflammatory phenotype in WAT (19). This induction of inflammation is the product of secreted cytokines such as TNF- $\alpha$, which promote ECM expression by adipocytes, further enhancing their inflammatory state and systemic insulin resistance. Surviving adipocytes, while increased in size, are mechanically restricted, thus compromising their capacity to incorporate fatty acids in the form of triglycerides. This process likely prompts spillover of fatty acids from inflamed, dysfunctional WAT adipocytes. In consequence, large lipid droplets accumulate in other tissues such as liver, muscle, and BAT, which participate in triglyceride clearance, thus compromising insulin sensitivity and decreasing energy expenditure due to reduced UCP1 $(9,35)$. The contention that myeloid-specific Asxl2 deletion protects BAT by preventing HFD-induced lipid spillover is supported by the simultaneous prevention of hepatic steatosis. In the absence of ASXL2, macrophages are less prone to inflammatory activation and ECM expression and as such, pathological WAT remodeling is limited which may prevent lipid spillover and restrict whitening of BAT.

On the other hand, BAT effectively incorporates triglycerides and metabolizes fatty acids, a process mediated by thermogenic activation involving UCP1 induction (9). Incorporation of triglycerides by BAT is enhanced in circumstances of increased dietary fat and restricted hepatic uptake, as occurs in steatosis. Thus, it is possible that prevention of HFD-induced BAT whitening by $A s x l 2^{4 L y s M}$ macrophages reflects arrest of dietary hyperlipidemia, thus limiting fatty acid incorporation.

Ultimately, however, catecholamines are central to maintenance of BAT morphology and function, raising the possibility that Asxl2 deletion prompts their production by myeloid lineage cells. Although norepinephrine abundance is approximately twice that of control in Asxl2 ${ }^{4 L y s M}$ BAT, tyrosine hydroxylase expression by WT BMMs and those lacking ASXL2 is undetectable, consistent with reports challenging the concept that alternatively activated macrophages produce catecholamines. The abundance of Maoa in the SVF of $A s x l 2^{4 L y s M}$ BAT indicates that accumulation of norepinephrine reflects its retarded degradation, which prevents whitening (17). Asxl2 deletion in myeloid cells reduces stimulated secretion of TNF- $\alpha$, which promotes Maoa expression (36), suggesting that Asxl2 ${ }^{4 L y s M}$ macrophages may preserve catecholamines by the cell's limited infiltration into BAT, regardless of weight gain and their diminished expression of proinflammatory cytokines. RNA-seq analysis revealed no difference in catecholamine-regulating protein expression by $A s x l 2^{4 L y s M}$ and $A s x l 2^{f l / f l}$ BMMs, supporting the concept that restricted macrophage infiltration, rather than diminished MAOA synthesis, is the dominant cause of BAT preservation. Confirmation of this conclusion, however, requires direct measurement of MAOA and SLC6A2 in BAT-residing macrophages, particularly those that are associated with sympathetic neurons, which are difficult to obtain in purity (17). Additionally, future studies will determine if prevention of macrophage accumulation in Asxl2 ${ }^{4 L y s M}$ BAT reflects modified proliferation of resident macrophages or dampened recruitment of circulating monocytes.

Because alternatively activated macrophages produce collagens and other matrix components, their ECM proteins may hypothetically contribute to the pathological remodeling of obese fat $(37,38)$. This being the case, macrophages that prevent obesity may express less ECM protein than control. In fact, the RNA-seq profile of $A s x l 2^{4 L y s M} \mathrm{BMMs}$ reveals a unique phenotype relative to classical alternatively activated cells in which collagen production is increased to enable tissue healing (39-41). Although crown-like structures typically contain M1-polarized macrophages, those associated with adipocyte fibrosis are predominantly M2 cells (42). The role that macrophage-secreted ECM proteins play in fibrotic remodeling of obesity-associated WAT is unknown but the paucity of these molecules, expressed by Asxl2 ${ }^{4 L y s M}$ macrophages, may contribute to their protection against diet-induced weight gain and insulin resistance.

Although UCP1 activity in WT BAT is robust at room temperature, mice lacking the uncoupling enzyme, in this circumstance, have normal body mass, indicating cold-associated compensatory mechanisms (i.e., non-UCP1) preventing weight gain $(43,44)$. At thermoneutrality, however, chow-fed $U c p 1^{-/-}$mice develop obesity (45) and in WT mice, HFD partially activates the enzyme $(45,46)$. Additionally, human BAT incorporates more glucose and releases more lactate than WAT in warm conditions (47). These observations indicate that HFD can stimulate BAT metabolism even at thermoneutrality and raise the possibility that preservation of BAT may diminish diet-induced weight gain in the absence of thermal stress. In fact, such appears to be the case regarding $A s x l 2^{4 L y s M}$ mice.

Collectively, these data highlight the role of ASXL2 in altering the gene expression profile of macrophages. Further, deletion of Asxl2 may protect adipose tissue homeostasis by locally influencing macrophage accumulation and function. Thus, the capacity of myeloid deletion of ASXL2 to prevent inflammatory remodeling of fat may represent a partnership of decreased recruitment of macrophages and suppression of their proinflammatory and ECM pathway genes.

To our knowledge, these findings are the first to identify ASXL2 as a master regulator of macrophage function to control systemic metabolic homeostasis. In addition, targeting ASXL2 in macrophages may represent a novel therapeutic strategy against obesity. Nevertheless, caution is warranted, as Asxl2 mutations are associated with macrocephaly and dysmorphic features (48) as well as hematopoietic malignancies. At least in mice, however, development of these leukemic disorders appears to require inactivation of the gene in hematopoietic stem cells that are not targeted by LysM-Cre (49). Thus, in contrast to mice lacking germline Asxl2, those in which the gene is absent only in myeloid lineage cells exhibit no splenomegaly or other evidence of leukemia. Furthermore, the fact that both LysM-Cre-mediated deletion and NP-associated Asxl2 siRNA prevent obesity adds further support for potential clinical relevance and indicates that postprandial arrest of the ETP protein is effective. Therefore, ASXL2 may have evolved to serve as a metabolic switch that determines macrophage responses to environmental stimuli such as dietary lipids.

\section{Methods}

Animals. LysM-Cre, Adipoq-Cre, Albumin-Cre (Alb-Cre), and Ucp1-/mice were obtained from The Jackson Laboratory. Bap $1^{f / f l}$ mice were generously provided by Genentech Inc. (50). All animals were housed in the animal care unit of Washington University School of Medicine, where they were maintained according to guidelines of the Association for Assessment and Accreditation of Laboratory Animal Care. 


\section{Table 1. List of primer sequences used for qPCR}

\begin{tabular}{|c|c|c|}
\hline Gene & Forward primer & Reverse primer \\
\hline Gapdh & 5'-TTCCACAGTCAGCCGCATCTTCTT-3' & 5'-CAGGCGCCCAATACGACCAAATC-3' \\
\hline Asxl2 & 5'-CTCCTCAAATGCAGGTGACA-3' & 5'-TTGCTTTGGGATCACTTGAG-3' \\
\hline Ucp1 & 5'-AGGCTTCCAGTACCATTAGGT-3' & 5'-CTGAGTGAGGCAAAGCTGATTT-3' \\
\hline Th & 5'-GTCTCAGAGCAGGATACCAAGC-3' & 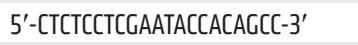 \\
\hline Maoa & 5'-GCCCAGTATCACAGGCCAC-3' & 5'-CGGGCTTCCAGAACCAAGA-3' \\
\hline Adgre1 & 5'-CTtTGGCTATGGGCTtCCAGtC-3' & 5'-GCAAGGAGGACAGAGTTTATCGTG-3 \\
\hline Fcgria & 5'-AGGTTCCTCAATGCCAAGTCA-3' & 5'-GCGACCTCCGAATCTGAACA-3' \\
\hline Cd68 & 5'-TGTCTGATCTTGCTAGGACCG-3' & 5'-GAGAGTAACGGCCTTTTTGTGA-3' \\
\hline 1116 & 5'-GTGCAAGTGTCTGAAGCAGC-3' & 5'-CAAAGGTTTGGAAGCAGCCC-3' \\
\hline Tnfa & 5'-CCTGTAGCCCACGTCGTAGC-3' & 5'-AGCAATGACTCCAAAGTAGACC-3' \\
\hline Colla1 & 5'-GCTCCTCTTAGGGGCCACT-3' & 5'-CCACGTCTCACCATTGGGG-3' \\
\hline Col3a & 5'-CTGTAACATCGAAACTGGGGAAA-3' & 5'-CСATAGCTCAACTCAAAACCACC-3' \\
\hline Col6a & 5'-CTGCTGCTACAAGCCTGCT-3' & 5'-CCCCATAAGGTTTCAGCCTCA-3' \\
\hline Col18a & 5'-GTGCCCATCGTCAACCTCAA-3' & 5'-GACATCTCTGCCGTCAAAAGAA-3' \\
\hline Itgva & 5'-CCGTGGACTTCTTCGAGCC-3' & 5'-CTGTTGAATCAAACTCAATGGGC-3' \\
\hline Mmp12 & 5'-CTGCTCCCATCAATCACAGTG-3' & 5'-AGTTGCTTCTAGCCCAAAGAAC-3' \\
\hline Mmp14 & 5'-СAGTATGGCTACCTACCTCCAG-3' & 5'-GCСTTGCCTGTCACTTGTAAA-3' \\
\hline$[x<12$ & 5'-AGTCAACTGCGCTGTCAATG-3' & 5'-ACTTTTTCACCGCCCTTCAG-3' \\
\hline Cxcl4 & 5'-AGGACATGGTCTTCACACACAC-3' & 5'-TTCTCGGCCTGTTGTTTCTG-3' \\
\hline Cxcl12 & 5'-TGCATCAGTGACGGTAAACCA-3' & 5'--TTCTTCAGCCGTCCAACAATC-3' \\
\hline Cxcl14 & 5'-GAACATCGTTATCGTCACCACC-3' & 5'-CGTTCCAGGCATTGTACCACT-3' \\
\hline Nlrp3 & 5'-TGCTCTTCACTGCTATCAAGCCCT-3' & 5'-ACAAGCCTTTGCTCCACACCCTAT-3' \\
\hline
\end{tabular}

Th, tyrosine hydroxylase.

Generation of Asxl2-deficient mice. Asxl2 ${ }^{\mathrm{tmla(EUCOMM)Wtsi}}$ (clone ID: HEPD0751_1_D05) embryonic stem cells were purchased from the European Conditional Mouse Consortium (EUCOMM) and were microinjected into B6(Cg)-Tyrc-2J/J blastocysts to generate chimeric mice at the Washington University Pathology/Immunology Micro-Injection Core. Highly chimeric mice were bred with $\mathrm{B} 6(\mathrm{Cg})-\mathrm{Tyrc}-2 \mathrm{~J} / \mathrm{J}$ females to select for germline transmission. To remove the LacZ-neomycin-resistance cassette, the mice were first crossed with transgenic mice expressing Flp recombinase under the control of the actin promoter (The Jackson Laboratory). The Flp transgene was removed by crossing the mice with WT C57BL6/J mice. Asx $x 2^{f /+}$ mice were intercrossed to generate $A s x l 2^{f / f l}$ mice. $A s x l 2^{f / f l}, A s x l 2^{A / W T}$, and $A s x l 2^{W T}$ littermate mice were genotyped by PCR with primers ASXL2-5'-arm (5'-CCCACACGCTCAGTCTCTACTCGC-3'), ASXL2-3'-arm (5'-TCTCCCTTCTTTGTTGCTGCCACC-3'), and LAR3 (5'-CAACGGGTTCTTCTGTTAGTCC-3') using the following parameters: $95^{\circ} \mathrm{C}$ for 3 minutes, followed by 35 cycles of $95^{\circ} \mathrm{C}$ for 30 seconds, $62^{\circ} \mathrm{C}$ for 30 seconds, and $72^{\circ} \mathrm{C}$ for 30 seconds, and then $72^{\circ} \mathrm{C}$ for 5 minutes. The WT allele was detected as a band at $828 \mathrm{bp}$, whereas the floxed allele was detected as a band at $642 \mathrm{bp}$. Asxl $2^{A / / f l}$ mice generated were subsequently crossed with LysM-Cre mice (The Jackson Laboratory), Adipoq-Cre, and Alb-Cre. After Cre recombination, the floxed and WT alleles were confirmed by PCR with primers (ASXL2-F, 5'-ACTTTCCTCAGACCATCAGCTTCC-3'; ASXL2-R, 5'-CCCCACGCCCCTCTCTCA-3'; and ASXL2-loxP, 5'-TGAACTGATGGCGAGCTCAGACC-3') using the same parameters as above. The WT allele was detected as a band at $320 \mathrm{bp}$, whereas the floxed allele was detected as a band at $378 \mathrm{bp}$.

RNA extraction and qPCR. RNA from cultured cells was isolated and purified using the RNeasy RNA purification kit (Qiagen); RLT lysis buffer was supplemented with $\beta$-mercaptoethanol (1\%). RNA from tis- sues was isolated using the TRIzol method. Purified RNA was treated with DNase I (Invitrogen) before reverse transcription. cDNA was synthesized from RNA $(1 \mu \mathrm{g})$ using the High-Capacity cDNA Reverse Transcription kit (Applied Biosystems). Real-time PCR was performed using the SYBR Green Master Mix kit and gene-specific primers. The qPCR reaction was performed on an ABI PRISM 7500 sequence detection system (Applied Biosystems). All reactions were performed in triplicate and relative mRNA levels were calculated by the comparative threshold cycle method using GAPDH as an internal control. Detailed sequences of the oligonucleotides used in qPCR analyses are listed in Table 1.

HFD feeding studies. Mice were housed in a pathogen-free barrier facility with unrestricted access to water and standard mouse chow containing $6 \%$ fat, which is designated as standard chow (chow) herein. For dietary intervention studies, mice were fed chow until age 8 weeks or 4 weeks and thereafter were randomized into groups that were fed either chow or an HFD (Research Diets, catalog D12492). Food intake and weight were monitored weekly. At the end of the study, serum was collected and tissues were snap-frozen in liquid nitrogen and stored at $-80^{\circ} \mathrm{C}$.

Body composition analysis. Body composition was measured using DXA with a Faxitron UltraFocus according to the manufacturer's instructions. Body fat mass and lean mass were measured between 2 pm and $3 \mathrm{pm}$.

Indirect calorimetry. Indirect calorimetry was performed as described previously (51). $\mathrm{O}_{2}$ consumption $\left(\mathrm{VO}_{2}\right)$ and $\mathrm{CO}_{2}$ production $\left(\mathrm{VCO}_{2}\right)$ were measured for 48 hours to determine energy expenditure. Mean relative $\mathrm{VO}_{2}$ (expressed in $\mathrm{mL} / \mathrm{kg} / \mathrm{min}$ ) and respiratory quotient (RQ; calculated as $\mathrm{VCO}_{2} / \mathrm{VO}_{2}$ ) were determined.

GTT and ITT. Glucose tolerance tests (GTTs) were performed on 16- to 20-week-old male mice by placing them in clean cages and starving 6 hours with free access to water. Mice were weighed and a small amount of blood was obtained from the lateral saphenous vein for baseline (time 0) glucose measurements. Mice were then injected intraperitoneally with $50 \%$ sterile dextrose ( $1 \mathrm{mg} / \mathrm{g}$ body weight). Tail blood glucose was determined at 15, 30, 60, and 120 minutes after challenge using a Bayer Contour glucometer. For insulin tolerance tests (ITTs), 16- to 20-week-old male mice were placed in clean cages without food but free access to water. Following a 6-hour fast, the mice were weighed and baseline glucose readings were taken using a Bayer Contour glucometer. Mice were injected intraperitoneally with human insulin (Humulin, Eli Lilly) at a dose of $0.5 \mathrm{U} / \mathrm{kg}$ body weight and blood glucose measured at 15, 30, 45, and 60 minutes afterward.

Thermoneutrality studies. Ucp1 $1^{-/}$mice were bred with Asxl2-Cre mice to generate $A s x l 2^{4 L y s M}$ on a $U c p 1^{-/-}$background. These mice, along with the controls ( $A s x l 2^{f / f l}, U c p 1^{--}$, and $A s x l 2^{4 L y s M}$ ) were housed at thermoneutrality. Mice were fed chow until age 8 weeks and thereafter were randomized into groups that were fed an HFD. Body weights were monitored weekly.

In vivo metabolic imaging. Mice were secured in a custom-designed acrylic restraining device and placed inside the field of view (FOV) of cross-calibrated Siemens Inveon PET/CT or Focus F220 scanners. A 30-minute dynamic positron emission tomography (PET) image acquisition was initiated immediately preceding a bolus injection of $\left[{ }^{18} \mathrm{~F}\right]$ fludeoxyglucose $(0.2-0.5 \mathrm{mCi})$ or $\left[{ }^{11} \mathrm{C}\right]$ palmitate via the tail vein. Dynamic images were reconstructed using the ordered subsets expectation maximization (OSEM) algorithm and 40 frames per imaging session. Images were analyzed by drawing regions of interest (ROIs) 
on BAT to ascertain in vivo measures of glucose (via fludeoxyglucose) and fatty acid (via $\left[{ }^{11} \mathrm{C}\right]$-palmitate) metabolism time course. Data derived from ROIs were normalized to standardized uptake values (SUV $=$ activity $\times$ [weight of mouse] $/$ [injected dose]) to account for differences in injected dose and weight of mice.

p5RHH-siRNA NP preparation. p5RHH peptide (provided by Genscript) was dissolved at $10 \mathrm{mM}$ in DNase-, RNase-, and protease-free sterile purified water (Cellgro) and stored in $10-\mu \mathrm{L}$ aliquots at $-80^{\circ} \mathrm{C}$ before use. The Cy5.5-labeled or nonlabeled siRNAs, scrambled siRNA (target sequence, GACGUAAACGGCCACAAGCC), and Asxl2 siRNA (target sequence, CCUAGGAGUUGCAGACUUG) were procured from Sigma-Aldrich, dissolved at $100 \mu \mathrm{M}$ in $1 \times$ siRNA buffer (Thermo Fisher Scientific), and stored in $10-\mu \mathrm{L}$ aliquots at $-80^{\circ} \mathrm{C}$ before use. The p5RHH-siRNA NPs were prepared by mixing equal volumes of the aforementioned p5RHH peptide and siRNA at a peptide/siRNA ratio of 100:1 in $200 \mu \mathrm{L}$ HBSS with $\mathrm{Ca}^{2+}$ and $\mathrm{Mg}^{2+}$ (Gibco and Life Technologies) and incubated at 10 minutes on ice before i.v. injection (26). For NP localization experiments, mice were sacrificed 24 hours after injection and tissues of interest were harvested and stored at $-80^{\circ} \mathrm{C}$. For body weight experiments, mice were i.v. injected with NPs twice per week after 3 weeks of HFD.

For in vitro experiments, the p5RHH-siRNA NPs were prepared by mixing equal volumes of the aforementioned p5RHH peptide and siRNA at a peptide/siRNA ratio of 100:1 in HBSS with $\mathrm{Ca}^{2+}$ and $\mathrm{Mg}^{2+}$ and incubated at $37^{\circ} \mathrm{C}$ for 40 minutes and then added to the macrophage culture.

Morphometric analysis of WAT. Epididymal WAT was fixed in $10 \%$ formalin for 2 days and transferred to $70 \%$ ethanol. Sections were stained with hematoxylin and eosin and images acquired using Image (NIH).

Fecal fat determination. Fecal fat content was determined gravimetrically as described previously (52). Briefly, $0.2 \mathrm{~g}$ of dried feces was solubilized in $1.6 \mathrm{~mL}$ water and homogenized using 1-mm glass beads. Feces were extracted in $6 \mathrm{~mL}$ chloroform/methanol (2:1) and the organic phase was transferred to a pre-weighed vial, dried under nitrogen, and reweighed to determine lipid mass.

Isolation of immune cells and flow cytometry. Murine epididymal WAT, iWAT, and BAT were harvested and digested with $0.1 \%$ collagenase type II (Sigma-Aldrich) at $37^{\circ} \mathrm{C}$ with shaking at $200 \mathrm{rpm}$ for 60 minutes. Digested tissues were filtered through a $100-\mu \mathrm{m}$ nylon mesh and centrifuged at $500 \mathrm{~g}$ for 5 minutes. Floating adipocytes were removed, and the SVF pellet was resuspended in red blood cell lysis buffer (ACK RBC Lysis Buffer). Recovered cells were washed and stained with Zombie UV (1:600; BioLegend) according to the manufacturer's protocol, followed by surface staining for flow cytometric analysis with fluorochrome-conjugated antibodies. The following antibodies were used: rat anti-mouse CD45-BUV395 (BD Horizon, clone 30-F11; 1:200), rat anti-mouse CD304-BV421 (BioLegend, clone 3E12; 1:300), rat anti-mouse CD86-BV605 (BioLegend, clone GL-1; 1:300), rat anti-mouse/human CD11b-BV650 (BioLegend, clone M1/70; 1:400), hamster anti-mouse CD11cBV711 (BioLegend, clone N418; 1:300), rat anti-mouse Ly6G-BV785 (BioLegend, clone 1A8; 1:300), rat anti-mouse F4/80-APC (BioLegend, clone BM8; 1:300), rat anti-mouse MHC-II-Alexa Fluor 700 (BioLegend, clone M5/114.15.2; 1:300), rat anti-mouse CD14-APC/ Cy7 (BioLegend, clone Sa14-2; 1:200), rat anti-mouse SiglecF-PE (BD Pharmingen, clone E50-2440; 1:400), rat anti-mouse CD206PE/Cy7 (BioLegend, clone C068C2; 1:200), mouse anti-mouse CD64-PE-Dazzle594 (BioLegend, clone X54-5/7.1; 1:300), and rat anti-mouse Ly6C-FITC (BioLegend, clone HK1.4; 1:300) in Brilliant Stain Buffer (BD Biosciences) containing $10 \mu \mathrm{g} / \mathrm{mL}$ Fc Block (purified unconjugated rat anti-mouse CD16/32; clone 2.4G2, BD Pharmingen). Cells were incubated in the staining cocktail on ice for 30 minutes. Stained cells were washed 3 times in $200 \mu \mathrm{L}$ FACS buffer (PBS containing 2.5\% heat-inactivated FBS and $2.5 \mathrm{mM}$ EDTA) before data acquisition on a BD X20 flow cytometer. Cells were counted using CountBright Absolute Counting Beads (Invitrogen) for flow cytometry according to the manufacturer's instructions. Cell frequencies were defined as percentage of the parent gate or as percentage of live cells, as indicated.

Lipolysis assay. For ex vivo lipolysis assay, $20 \mathrm{mg}$ of BAT was collected and cultured in $200 \mu \mathrm{L}$ of low-glucose DMEM supplemented with $2 \%$ fatty acid-free BSA with or without $10 \mu \mathrm{M}$ isoproterenol in a 96-well plate for 60 minutes at $37^{\circ} \mathrm{C}$. Media were collected and glycerol release assays (MilliporeSigma) were performed per the manufacturer's protocol. Tissue samples were harvested for protein quantification using Bradford reagent.

Immunohistochemical staining. Paraffin sections $(5 \mu \mathrm{m})$ were rehydrated and treated with $0.3 \%$ hydrogen peroxide in methanol for 15 minutes to suppress the endogenous peroxidase activity. Antigen retrieval was achieved by microwaving the sections in $10 \mathrm{mM}$ citrate buffer for 10 minutes followed by gradual cooling to room temperature. Sections were incubated overnight at $4^{\circ} \mathrm{C}$. Immunostaining was detected using the Histostain-SP Broad Spectrum (DAB) kit (Thermo Fisher Scientific, 95-9643).

Hypoxyprobe labeling. HFD-fed control or Asxl2 $2^{4 L y s M}$ mice were injected intraperitoneally with $60 \mathrm{mg} / \mathrm{kg}$ hypoxyprobe-1 (pimonidazole $\mathrm{HCl}$, Hypoxyprobe, Inc.) in PBS. Gonadal WAT was isolated 75 minutes later and fixed overnight at $4^{\circ} \mathrm{C}$. Mice that did not receive hypoxyprobe were analyzed in parallel to serve as a negative control for hypoxyprobe antibody specificity. Detection of hypoxyprobe binding was performed using the Hypoxyprobe-1 Plus Kit (Hypoxyprobe, Inc.).

Macrophage isolation. Primary BMMs were prepared as described previously (53) with slight modification. Marrow was extracted from femora and tibiae of 6- to 8-week-old mice with $\alpha$-MEM and cultured in $\alpha$-MEM containing $10 \%$ inactivated FBS, $100 \mathrm{IU} / \mathrm{mL}$ penicillin, and $100 \mu \mathrm{g} / \mathrm{mL}$ streptomycin ( $\alpha-10$ medium) with conditioned media (1:10) from CMG12-14 cells (murine M-CSF-producing cell line; ref. 54) on plastic petri dishes. Cells were incubated at $37^{\circ} \mathrm{C}$ in $6 \% \mathrm{CO}_{2}$ for 3 days and then washed with PBS and lifted with $1 \times$ trypsin/EDTA in PBS.

NLRP3 inflammasome activation. Cultured BMMs were primed for 4 hours with LPS (100 ng/mL; Sigma-Aldrich) or TNF- $\alpha$ (10 ng/ $\mathrm{mL})$ alone, and then exposed to nigericin $(15 \mu \mathrm{M})$ for an additional hour to activate the NLRP3 inflammasome. Media were collected for IL-1 $\beta$ ELISA and cells harvested for either Western blot or gene expression analysis. To study NLRP3 inflammasome activation based on caspase- 1 activity, cells were cultured on coverslips and primed with LPS for 4 hours. Cells were stimulated with nigericin for 30 minutes followed by incubation with FLICA FAM-YVAD-FMK probe (ImmunoChemistry Technology LLC) for an additional 30 minutes and analyzed by fluorescence microscopy.

Western blot analysis and immunoprecipitation. Cultured cells were washed twice with ice-cold PBS and lysed in radioimmunoprecipitation assay (RIPA) buffer containing $20 \mathrm{mM}$ Tris- $\mathrm{HCl}, \mathrm{pH} 7.5$, 
150 mM NaCl, 1 mM EDTA, 1 mM EGTA, 1\% Triton X-100, 2.5 mM sodium pyrophosphate, $1 \mathrm{mM} \beta$-glycerophosphate, $1 \mathrm{mM} \mathrm{Na}_{3} \mathrm{VO}_{4}, 1$ $\mathrm{mM} \mathrm{NaF}$, and $1 \times$ protease inhibitor mixture (Roche Applied Science). Total cell lysates were prepared by homogenizing the tissue in TNET lysis buffer consisting of $1 \%$ Triton X-100, $150 \mathrm{mM} \mathrm{NaCl}, 50 \mathrm{mM}$ Tris pH 7.4, and 2 mM EDTA supplemented with protease and phosphatase inhibitors. After incubation on ice for 10 minutes, cell lysates were clarified by centrifugation at $21,000 \mathrm{~g}$ for 10 minutes. Total lysates ( $40 \mu \mathrm{g}$ protein) were resolved by $8 \%-12 \%$ sodium dodecyl sulfate polyacrylamide gel electrophoresis and transferred onto PVDF membranes. Filters were blocked in $0.1 \%$ casein in PBS for 1 hour and incubated with primary antibodies at $4^{\circ} \mathrm{C}$ overnight followed by probing with fluorescently labeled secondary antibodies (Jackson ImmunoResearch Laboratories). Proteins were detected with the Odyssey Infrared Imaging System (LI-COR Biosciences).

RNA-seq library preparation and sequencing. Total RNA integrity was determined using an Agilent Bioanalyzer or 4200 Tapestation. Library preparation was performed with $10 \mu \mathrm{g}$ of total RNA with a Bioanalyzer RIN score greater than 8.0. Ribosomal RNA was removed by poly-A selection using oligo-dT beads (mRNA Direct kit, Life Technologies). mRNA was then fragmented in buffer containing $40 \mathrm{mM}$ Tris acetate $\mathrm{pH} 8.2,100 \mathrm{mM}$ potassium acetate, and $30 \mathrm{mM}$ magnesium acetate and heated to $94^{\circ} \mathrm{C}$ for 150 seconds. mRNA was reverse transcribed to yield cDNA using SuperScript III RT enzyme (Life Technologies, per the manufacturer's instructions) and random hexamers. A second-strand reaction was performed to yield double-stranded cDNA. cDNA was blunt ended, had an A base added to the $3^{\prime}$ ends, and then had Illumina sequencing adapters ligated to the ends. Ligated fragments were then amplified for 12 cycles using primers incorporating unique index tags. Fragments were sequenced on an Illumina HiSeq 2500 using single reads extending 50 bases.

RNA-seq data acquisition, quality control, and processing. RNAseq reads were aligned to the GRCm38.76 assembly from Ensembl with STAR version 2.0.4b (55). Gene counts were derived from the number of uniquely aligned unambiguous reads by Subread:featureCount version 1.4.5. Transcript counts were produced by Sailfish version 0.6.3. Sequencing performance was assessed for total number of aligned reads, total number of uniquely aligned reads, genes and transcripts detected, ribosomal fraction known junction saturation, and read distribution over known gene models with $\mathrm{RSeQC}$ version 2.3. All gene-level and transcript counts were then imported into the R/Bioconductor package EdgeR and TMM normalized to adjust for differences in library size. Genes or transcripts not expressed in any sample were excluded from further analysis. Performance of the samples was assessed with a Spearman correlation matrix and multidimensional scaling plots. Generalized linear models with robust dispersion estimates were created to test for gene/transcript level differential expression. The fit of the trended and tagwise dispersion estimates were then plotted to confirm proper fit of the observed mean-to-variance relationship where the tagwise dispersions are equivalent to the biological coefficients of variation of each gene. Differentially expressed genes and transcripts were then filtered for FDR-adjusted $P$ values less than or equal to 0.05 .

To enhance the biological interpretation of the large set of transcripts, grouping of genes/transcripts based on functional similarity was achieved using the R/Bioconductor packages GAGE and Path- view. GAGE and Pathview were also used to perform pathway maps on known signaling and metabolism pathways curated by KEGG. The raw data have been deposited to the NCBI's Gene Expression Omnibus (GEO) repository (GSE144180).

Statistics. Statistical significance was determined using unpaired, 2-tailed Student's $t$ test, or 1-way or 2-way ANOVA with the Holm-Sidak multiple-comparisons test. Data are expressed as mean \pm SD. ${ }^{*} P<$ $0.05,{ }^{* *} P<0.01$, and ${ }^{* *} P<0.001$ indicate the level of significance in all experiments.

Study approval. All animal experimentation was approved by the Animal Studies Committee of Washington University School of Medicine.

\section{Author contributions}

WZ, NR, JRB, KIS, GM, SAW, NAA, and SLT were responsible for conceptualization. WZ, NR, JRB, JRM, JWW, KIS, SAW, NAA, and SLT developed methodology. SLT validated the data. RDH performed formal analysis. WZ, NR, JRB, YL, JWW, YA, HP, TAP, EPN, and KIS performed investigations. $\mathrm{AD}, \mathrm{SAW}$, and NAA acquired resources. RDH, SAW, NAA, and SLT curated data. WZ, NR, and SLT wrote the original draft of the manuscript, which GM, KIS, SAW, and NAA reviewed and edited. NOD, GJR, NAA, and SLT supervised the study. SLT administered the project and acquired funding. WZ and NR are sequentially listed as first authors. Our decision in this regard was based on the following: WZ developed the hypothesis that myeloid deletion of ASXL2 would prevent diet-induced weight gain. He also made the initial discovery that his hypothesis was correct and that the mechanism involved preservation of brown adipose tissue. NR discovered the role of altered energy expenditure and differences in processing catecholamines and suggested and supervised RNA-seq analysis of BMMs. She also brought the BAP1-based experiments to the study. Given that WZ's contribution was initiating the project and establishing key observations, he has been listed first.

\section{Acknowledgments}

This study was supported by the following grants from the NIH: K99 HL1388163 (to JWW), AR064755 (to GM), AR068972 (to GM), AR070975 (to GM), HL38180 (to NOD), DK56260 (to NOD), P30 DK52574 (to NOD), P41 EB025815 (to KIS), HL073646 (to SAW), DK102691 (to SAW), AI019653 (to GJR), DK109668 (to GJR), DK056341 (to NAA), AR046523 (to SLT), DK111389 (to SLT), and P30 AR074992 (to SLT and GM). JRB is supported by the Physician-Scientist Training Program at Washington University School of Medicine and the Children's Discovery Institute. We thank the Cyclotron and the Preclinical PET/CT Imaging facilities at Mallinckrodt Institute of Radiology (MIR) for production of radiopharmaceuticals and imaging studies.

Address correspondence to: Steven L. Teitelbaum, Campus Box 8118, 660 South Euclid Avenue, St. Louis, Missouri 63110, USA. Phone: 314.454.8463; Email: teitelbs@wustl.edu.

JWW's present address is: Department of Integrative Biology and Physiology, University of Minnesota Medical School, Minneapolis, Minnesota, USA. 
1. Wellen KE, Hotamisligil GS. Inflammation, stress, and diabetes. J Clin Invest. 2005;115(5):1111-1119.

2. Lumeng CN, Bodzin JL, Saltiel AR. Obesity induces a phenotypic switch in adipose tissue macrophage polarization. JClin Invest. 2007;117(1):175-184.

3. Patsouris D, Li PP, Thapar D, Chapman J, Olefsky JM, Neels JG. Ablation of CD11c-positive cells normalizes insulin sensitivity in obese insulin resistant animals. Cell Metab. 2008;8(4):301-309.

4. Izawa T, et al. ASXL2 regulates glucose, lipid, and skeletal homeostasis. Cell Rep. 2015;11(10):1625-1637.

5. Lee KY, et al. Lessons on conditional gene targeting in mouse adipose tissue. Diabetes. 2013;62(3):864-874

6. Chen EY, et al. Enrichr: interactive and collaborative HTML5 gene list enrichment analysis tool. BMC Bioinformatics. 2013;14:128

7. Kuleshov MV, et al. Enrichr: a comprehensive gene set enrichment analysis web server 2016 update. Nucleic Acids Res. 2016;44(W1):W90-W97.

8. Chen Y, et al. Exosomal microRNA miR-92a concentration in serum reflects human brown fat activity. Nat Commun. 2016;7:11420.

9. Bartelt A, et al. Brown adipose tissue activity controls triglyceride clearance. Nat Med. 2011;17(2):200-205.

10. Shimizu I, et al. Vascular rarefaction mediates whitening of brown fat in obesity. JClin Invest. 2014;124(5):2099-2112.

11. Blondin DP, et al. Dietary fatty acid metabolism of brown adipose tissue in cold-acclimated men. Nat Commun. 2017;8:14146.

12. Yoneshiro T, et al. Recruited brown adipose tissue as an antiobesity agent in humans. JClin Invest. 2013;123(8):3404-3408.

13. Eisinger K, Liebisch G, Schmitz G, Aslanidis C, Krautbauer S, Buechler C. Lipidomic analysis of serum from high fat diet induced obese mice. Int JMol Sci. 2014;15(2):2991-3002.

14. Qiu Y, et al. Eosinophils and type 2 cytokine signaling in macrophages orchestrate development of functional beige fat. Cell. 2014;157(6):1292-1308

15. Fischer K, et al. Alternatively activated macrophages do not synthesize catecholamines or contribute to adipose tissue adaptive thermogenesis. Nat Med. 2017;23(5):623-630.

16. Camell CD, et al. Inflammasome-driven catecholamine catabolism in macrophages blunts lipolysis during ageing. Nature. 2017;550(7674):119-123.

17. Pirzgalska RM, et al. Sympathetic neuron-associated macrophages contribute to obesity by importing and metabolizing norepinephrine. Nat Med. 2017;23(11):1309-1318.

18. Wu D, et al. Eosinophils sustain adipose alternatively activated macrophages associated with glucose homeostasis. Science. 2011;332(6026):243-247.

19. Sun K, Kusminski CM, Scherer PE. Adipose tissue remodeling and obesity. JClin Invest. 2011;121(6):2094-2101.

20. Saltiel AR, Olefsky JM. Inflammatory mechanisms linking obesity and metabolic disease. JClin Invest. 2017;127(1):1-4.

21. Alippe $\mathrm{Y}$, et al. Bone matrix components activate the NLRP3 inflammasome and promote osteoclast differentiation. Sci Rep. 2017;7(1):6630.

22. Strissel KJ, et al. Adipocyte death, adipose tissue remodeling, and obesity complications. Diabetes. 2007;56(12):2910-2918.

23. Divoux A, et al. Fibrosis in human adipose tissue: composition, distribution, and link with lipid metabolism and fat mass loss. Diabetes. 2010;59(11):2817-2825.

24. Daou S, et al. The BAP1/ASXL2 histone H2A deubiquitinase complex regulates cell proliferation and is disrupted in cancer. J Biol Chem. 2015;290(48):28643-28663.

25. Hou KK, Pan H, Lanza GM, Wickline SA. Melittin derived peptides for nanoparticle based siRNA transfection. Biomaterials. 2013;34(12):3110-3119.

26. Hou KK, Pan H, Ratner L, Schlesinger PH, Wickline SA. Mechanisms of nanoparticle-mediated siRNA transfection by melittin-derived peptides. ACS Nano. 2013;7(10):8605-8615.

27. Hou KK, Pan H, Schlesinger PH, Wickline SA. A role for peptides in overcoming endosomal entrapment in siRNA delivery - A focus on melittin. Biotechnol Adv. 2015;33(6 pt 1):931-940.

28. Zhang CY, Dong X, Gao J, Lin W, Liu Z, Wang Z. Nanoparticle-induced neutrophil apoptosis increases survival in sepsis and alleviates neurological damage in stroke. Sci $A d v$. 2019;5(11):eaax7964.

29. Zhou HF, et al. Peptide-siRNA nanocomplexes targeting NF- $\mathrm{\kappa B}$ subunit $\mathrm{p} 65$ suppress nascent experimental arthritis. J Clin Invest. 2014;124(10):4363-4374.

30. Yan $\mathrm{H}$, et al. Suppression of NF- $\mathrm{KB}$ activity via nanoparticle-based siRNA delivery alters early cartilage responses to injury. Proc Natl Acad Sci U S A. 2016;113(41):E6199-E6208.

31. Dehghan M, et al. Associations of fats and carbohydrate intake with cardiovascular disease and mortality in 18 countries from five continents (PURE): a prospective cohort study. Lancet 2017;390(10107):2050-2062.

32. Roberts MN, et al. A ketogenic diet extends longevity and healthspan in adult mice. Cell Metab. 2017;26(3):539-546.e5.

33. Baughman JM, et al. NeuCode proteomics reveals Bap1 regulation of metabolism. Cell Rep. 2016;16(2):583-595

34. Hatori M, et al. Time-restricted feeding without reducing caloric intake prevents metabolic diseases in mice fed a high-fat diet. Cell Metab. 2012;15(6):848-860.

35. Gao X, Salomon C, Freeman DJ. Extracellular vesicles from adipose tissue-a potential role in obesity and type 2 diabetes? Front Endocrinol (Lausanne). 2017;8:202.

36. Gupta V, Khan AA, Sasi BK, Mahapatra NR. Molecular mechanism of monoamine oxidase A gene regulation under inflammation and ischemia-like conditions: key roles of the transcription factors GATA2, Sp1 and TBP. J Neurochem. 2015;134(1):21-38.

37. Cai J, Li B, Liu K, Li G, Lu F. Macrophage infiltration regulates the adipose ECM reconstruction and the fibrosis process after fat grafting. Biochem Biophys Res Commun. 2017;490(2):560-566.

38. Schnoor M, et al. Production of type VI collagen by human macrophages: a new dimension in macrophage functional heterogeneity. J Immunol. 2008;180(8):5707-5719.

39. Gordon S. Alternative activation of macrophages. Nat Rev Immunol. 2003;3(1):23-35.

40. Wynn TA. Fibrotic disease and the $\mathrm{T}(\mathrm{H}) 1 / \mathrm{T}(\mathrm{H}) 2$ paradigm. Nat Rev Immunol. 2004;4(8):583-594.

41. Lech M, Anders HJ. Macrophages and fibrosis: How resident and infiltrating mononuclear phagocytes orchestrate all phases of tissue injury and repair. Biochim Biophys Acta. 2013;1832(7):989-997.

42. Spencer M, et al. Adipose tissue macrophages in insulin-resistant subjects are associated with collagen VI and fibrosis and demonstrate alternative activation. Am J Physiol Endocrinol Metab. 2010;299(6):E1016-E1027.

43. Lowell BB, Spiegelman BM. Towards a molecular understanding of adaptive thermogenesis. Nature. 2000;404(6778):652-660.

44. Golozoubova V, Gullberg H, Matthias A, Cannon B, Vennström B, Nedergaard J. Depressed thermogenesis but competent brown adipose tissue recruitment in mice devoid of all hormone-binding thyroid hormone receptors. Mol Endocrinol. 2004;18(2):384-401.

45. Feldmann HM, Golozoubova V, Cannon B, Nedergaard J. UCP1 ablation induces obesity and abolishes diet-induced thermogenesis in mice exempt from thermal stress by living at thermoneutrality. Cell Metab. 2009;9(2):203-209.

46. Kazak L, et al. UCP1 deficiency causes brown fat respiratory chain depletion and sensitizes mitochondria to calcium overload-induced dysfunction. Proc Natl Acad Sci US A. 2017;114(30):7981-7986.

47. Weir $\mathrm{G}$, et al. Substantial metabolic activity of human brown adipose tissue during warm conditions and cold-induced lipolysis of local triglycerides. Cell Metab. 2018;27(6):1348-1355.e4

48. Shashi $\mathrm{V}$, et al. De novo truncating variants in ASXL2 are associated with a unique and recognizable clinical phenotype. Am J Hum Genet. 2016;99(4):991-999.

49. Li J, et al. Loss of Asxl2 leads to myeloid malignancies in mice. Nat Commun. 2017;8:15456.

50. Dey A, et al. Loss of the tumor suppressor BAP1 causes myeloid transformation. Science. 2012;337(6101):1541-1546.

51. Funai K, et al. Muscle lipogenesis balances insulin sensitivity and strength through calcium signaling. J Clin Invest. 2013;123(3):1229-1240.

52. Newberry EP, Xie Y, Kennedy SM, Luo J, Davidson NO. Protection against Western diet-induced obesity and hepatic steatosis in liver fatty acid-binding protein knockout mice. Hepatology. 2006;44(5):1191-1205.

53. Lam J, Takeshita S, Barker JE, Kanagawa O, Ross FP, Teitelbaum SL. TNF-alpha induces osteoclastogenesis by direct stimulation of macrophages exposed to permissive levels of RANK ligand. J Clin Invest. 2000;106(12):1481-1488

54. Takeshita S, Kaji K, Kudo A. Identification and characterization of the new osteoclast progenitor with macrophage phenotypes being able to differentiate into mature osteoclasts. J Bone Miner Res. 2000;(8):1477-1488.

55. Dobin A, et al. STAR: ultrafast universal RNA-seq aligner. Bioinformatics. 2013;29(1):15-21. 